\title{
Stronger Lempel-Ziv Based Compressed Text Indexing*
}

\author{
Diego Arroyuelo ${ }^{\star \star \star}$, Gonzalo Navarro ${ }^{1 \star \star \star}$, and Kunihiko Sadakane ${ }^{2 \dagger}$ \\ 1 Dept. of Computer Science, Universidad de Chile, \\ Blanco Encalada 2120, Santiago, Chile. \\ \{darroyue, gnavarro\}@dcc.uchile.cl \\ 2 Dept. of Computer Science and Communication Engineering, Kyushu University, \\ Hakozaki 6-10-1, Higashi-ku, Fukuoka 812-8581, Japan. \\ sada@csce.kyushu-u.ac.jp
}

\begin{abstract}
Given a text $T[1 . . u]$ over an alphabet of size $\sigma$, the full-text search problem consists in finding the occ occurrences of a given pattern $P[1 . . m]$ in $T$. In indexed text searching we build an index on $T$ to improve the search time, yet increasing the space requirement. The current trend in indexed text searching is that of compressed full-text self-indices, which replace the text with a more space-efficient representation of it, at the same time providing indexed access to the text. Thus, we can provide efficient access within compressed space.

The LZ-index of Navarro is a compressed full-text self-index able to represent $T$ using $4 u H_{k}(T)+$ $o(u \log \sigma)$ bits of space, where $H_{k}(T)$ denotes the $k$-th order empirical entropy of $T$, for any $k=$ $o\left(\log _{\sigma} u\right)$. This space is about four times the compressed text size. It can locate all the occ occurrences of a pattern $P$ in $T$ in $O\left(m^{3} \log \sigma+(m+o c c) \log u\right)$ worst-case time. Despite this index has shown to be very competitive in practice, the $O\left(\mathrm{~m}^{3} \log \sigma\right)$ term can be excessive for long patterns. Also, the factor 4 in its space complexity makes it larger than other state-of-the-art alternatives.

In this paper we present stronger Lempel-Ziv based indices, improving the overall performance of the LZ-index. We achieve indices requiring $(2+\epsilon) u H_{k}(T)+o(u \log \sigma)$ bits of space, for any constant $\epsilon>0$, which makes our indices the smallest existing LZ-indices. We simultaneously improve the search time to $O\left(m^{2}+(m+o c c) \log u\right)$, which makes our indices very competitive with state-of-the-art alternatives. Our indices support displaying of any text substring of length $\ell$ in optimal $O\left(\ell / \log _{\sigma} u\right)$ time. In addition, we show how the space can be squeezed to $(1+\epsilon) u H_{k}(T)+o(u \log \sigma)$ to obtain a structure with $O\left(m^{2}\right)$ average search time for $m \geqslant 2 \log _{\sigma} u$. Alternatively, the search time of LZ-indices can be improved to $O((m+o c c) \log u)$ with $(3+\epsilon) u H_{k}(T)+o(u \log \sigma)$ bits of space, which is about half of the space needed by other Lempel-Ziv-based indices achieving the same search time. Overall our indices stand out as a very attractive alternative for space-efficient indexed text searching.
\end{abstract}

\section{Introduction}

Text searching is a classical problem in Computer Science. Given a sequence of symbols T[1..u $]$ (the text) over an alphabet $\Sigma=\{1, \ldots, \sigma\}$, and given another (short) sequence $P[1 . . m]$ (the search pattern) over $\Sigma$, the full-text search problem consists in finding all the occ occurrences of $P$ in $T$. There exist three typical kind of queries, namely:

- Existential queries: Operation exists $(P)$ tells us whether pattern $P$ exists in $T$ or not.

- Cardinality queries: Operation count $(P)$ counts the number of occurrences of pattern $P$ in $T$.

- Locating queries: Operation locate $(P)$ reports the starting positions of the occ occurrences of pattern $P$ in $T$.

* A preliminary version of this paper appeared in Proc. of the 17th Annual Symposium on Combinatorial Pattern Matching (CPM 2006), Volume 4009 of LNCS, 2006.

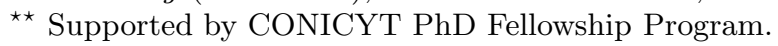

$\star \star \star$ Supported in part by Fondecyt Grant 1-080019.

$\dagger$ Supported in part by the Grant-in-Aid of the Ministry of Education, Science, Sports and Culture of Japan. 
With the huge amount of text data available nowadays, the full-text search problem plays a fundamental role in modern computer applications, which include text databases in general. Unlike word-based text searching, we wish to find any text substring, not only whole words or phrases. This has applications in texts where the concept of word is not well defined (e.g. Oriental languages), or texts where words do not exist at all (e.g., DNA, protein, and MIDI pitch sequences, program code, etc.). We assume that the text is large and known in advance to queries, and we need to perform several queries on it. Therefore, we can construct an index on the text, which is a data structure allowing efficient access to the pattern occurrences, yet increasing the space requirement.

Classical full-text indices, like suffix trees [1] and suffix arrays [27], have the problem of a high space requirement: they require $O(u \log u)$ and $u \log u$ bits respectively, which in practice is about 10-20 and 4 times the text size respectively, apart from the text itself. Thus, we can have large texts which fit into main memory, but whose corresponding suffix tree (or array) cannot be handled in main memory. Using secondary storage for the indices is several orders of magnitude slower, so one looks for ways to reduce their size, with the main motivation of maintaining the indices of very large texts entirely in main memory. Therefore, we seek to to provide fast access to the text using as little space as possible. The modern trend is to use the compressibility of the text to reduce the space of the index. In recent years there has been much research on compressed text databases, focusing on techniques to represent the text and the index using little space, yet permitting efficient text searching [35].

\subsection{Compressed Full-Text Self-Indexing}

To provide fast access to the text using little space, the current trend is to use compressed full-text self-indices. A full-text self-index allows one to retrieve any part of the text without storing the text itself, and in addition provides search capabilities on the text. A full-text compressed self-index is one whose space requirement is proportional to the compressed text size (e.g., $O\left(u H_{k}(T)\right)$ bits $\left.^{3}\right)$. Then a compressed full-text self-index replaces the text with a more space-efficient representation of it, which at the same time provides indexed access to the text [35, 12]. Taking space proportional to the compressed text, replacing it, and providing efficient indexed access to it is an unprecedented breakthrough in text indexing and compression.

As compressed full-text self-indices replace the text, we are also interested in operation:

- extract $(i, j)$, which decompresses the substring $T[i . . j]$, for any text positions $i \leqslant j$.

Note that compressed self-indices can be seen from two points of view: as full-text indices compressing the text, or as compression techniques allowing efficient indexed full-text searching.

The main types of compressed self-indices [35] are Compressed Suffix Arrays [18, 39], indices based on backward search [12] (which are alternative ways to compress suffix arrays, known as the FM-index family), and the indices based on the Lempel-Ziv compression algorithm [42] (LZ-indices for short) $[23,34,12,38]$. In this paper we are interested in LZ-indices, since they have shown to be very effective in practice for locating occurrences and extracting text, outperforming other compressed indices. Compressed indices based on suffix arrays store extra non-compressible information to carry out these tasks, whereas the extra data stored by LZ-indices is compressible. Therefore, when the texts are highly compressible, LZ-indices can be smaller and faster than alternative indices; and in other cases they offer very attractive space/time trade-offs. What characterizes the

\footnotetext{
${ }^{3} u H_{k}(T)$, the $k$-th order empirical entropy of $T$, is a lower bound to the number of bits used to represent $T$ by any $k$-th order compressor. See Section 2.2 for more details.
} 
particular niche of LZ-indices is the $O\left(u H_{k}(T)\right)$ space combined with $O(\log u)$ time per located occurrence.

Historically, the first compressed index based on Lempel-Ziv compression was that of Kärkkäinen and Ukkonen [23,22], which has a locating time $O\left(m^{2}+(m+o c c) \log u\right)$ and a space requirement of $O\left(u H_{k}(T)\right)$ bits, plus the text (as it is needed to operate) [35]. Navarro's LZ-index [34, 33], on the other hand, is a compressed full-text self-index based on the Lempel-Ziv 1978 [42] (LZ78 for short) parsing of the text. See Section 2.3 for a description of the LZ78 compression algorithm. The LZ-index takes about 4 times the size of the compressed text, i.e. $4 u H_{k}(T)+o(u \log \sigma)$ bits, for any $k=o\left(\log _{\sigma} u\right)[24,12]$, and answers queries in $O\left(m^{3} \log \sigma+(m+o c c) \log u\right)$ worst-case time. The index also replaces the text (i.e., it is a self-index): it can display a text context of length $\ell$ around an occurrence found (and in fact any sequence of LZ78 phrases) in $O(\ell \log \sigma)$ time, or obtain the whole text in time $O(u \log \sigma)$. The index is built in $O(u \log \sigma)$ time.

Despite this index has shown to be very competitive in practice [34,33], the $O\left(m^{3} \log \sigma\right)$ term in the search time makes it appropriate only for short patterns. Besides, in practice the space requirement of the LZ-index is relatively large compared with competing schemes: 1.2-1.6 times the text size versus 0.6-0.7 and 0.3-0.8 times the text size of the Compressed Suffix Array (CSA) [39] and the FM-index [12], respectively. Yet, the LZ-index is faster to locate and to display the context of an occurrence. Fast displaying of text substrings is very important in self-indices, as the text is not available otherwise. So the challenge is: can we reduce the space requirement of the LZ-index while retaining its good features?

\subsection{Our Contribution}

In this paper we study how to reduce by half the space requirement of the LZ-index, while at the same time improving its time complexities. The result is an attractive alternative to current state of the art in compressed self-indexing.

In a first approach (Section 4), we compress one of the data structures conforming the original LZ-index by using an approach which is, in some sense, related to the compression of suffix arrays $[18,39]$. In a second approach (Section 5) we combine the balanced parentheses representation of Munro and Raman [32] of the LZ78 trie with the xbw transform of Ferragina et al. [11], whose powerful operations are useful for the LZ-index search algorithm.

Despite these approaches are very different, if $\sigma=\Theta(\operatorname{polylog}(u))$ (representing moderate-size alphabets, which are very common in practice) in both cases we achieve $(2+\epsilon) u H_{k}(T)+o(u \log \sigma)$ bits of space, for any constant $\epsilon>0$, and simultaneously improve the original worst-case search time to $O\left(m^{2}+(m+o c c) \log u\right)$, thus achieving the same search time as the index of Kärkkäinen and Ukkonen [23], yet ours are much smaller and do not need the text to operate. In both cases we also present a version requiring $(1+\epsilon) u H_{k}(T)+o(u \log \sigma)$ bits, with average search time $O\left(m^{2}\right)$ if $m \geqslant 2 \log _{\sigma} u$. This space can get as close as desired to the optimal $u H_{k}(T)$ under the $k$-th order entropy model. The worst-case time for extracting any text substring of length $\ell$ is also improved to the optimal $O\left(\ell / \log _{\sigma} u\right)$ for all of our indices.

Just as for the original LZ-index, our data structures require $O\left(u H_{k}(T)\right)$ bits and spend $O(\log u)$ time per occurrence reported, if $\sigma=\Theta(\operatorname{polylog}(u))$. This fast locating is the strongest point of our structure. Other data structures achieving the same or better complexity for locating occurrences either are of size $O\left(u H_{0}(T)\right)$ bits plus a non-negligible extra space of $O(u \log \log \sigma)$ [39], or they achieve this locating time for constant-size alphabets [12]. Finally, the CSA of Grossi, Gupta, and Vitter [17] requires $\epsilon^{-1} u H_{k}(T)+o(u \log \sigma)$ bits of space, with a locating time of $O\left((\log u)^{\frac{\epsilon}{1-\epsilon}}\right.$ 
$\left.(\log \sigma)^{\frac{1-2 \epsilon}{1-\epsilon}}\right)$ per occurrence, after a counting time of $O\left(\frac{m}{\log _{\sigma} u}+(\log u)^{\frac{1+\epsilon}{1-\epsilon}}(\log \sigma)^{\frac{1-3 \epsilon}{1-\epsilon}}\right)$, where $0<$ $\epsilon<1 / 2$ is a constant. When $\epsilon$ approaches $1 / 2$, the space requirement approaches (from above) $2 u H_{k}(T)+o(u \log \sigma)$ bits, with a counting time of $O\left(\frac{m}{\log _{\sigma} u}+\frac{\log ^{3} u}{\log \sigma}\right)$ and a locating time per occurrence of still $\omega(\log u)$.

In Table 1 we summarize the space and time complexities of some existing compressed selfindices (other less competitive ones are ignored [35]). Total locate times in the table are after counting the pattern occurrences. For counting the number of occurrences of $P$ in $T$, our data structures are not competitive against schemes requiring about the same space $[17,13]$. Yet, in many practical situations, it is necessary to report the occurrence positions, as well as displaying their contexts and extracting (or uncompressing) any text substring. In this aspect, as explained, our LZ-indices are superior.

A new LZ-index, the Inverted LZ-index (ILZI for short) [38], has appeared independently and simultaneously with our work [4]. The ILZI is faster than our data structures since it can report the pattern occurrences in $O((m+o c c) \log u)$ time, but at the price of a higher space requirement: $(5+\epsilon) u H_{k}(T)+o(u \log \sigma)$ bits. However, in this paper we also show that the same reporting time $O((m+o c c) \log u)$ can be obtained with a significatively smaller LZ-index requiring $(3+\epsilon) u H_{k}(T)+$ $o(u \log \sigma)$ bits of space. In practice, our LZ-indices and the ILZI are comparable.

Table 1. Comparison of our LZ-index with alternative compressed self-indices. We assume $\sigma=O(\operatorname{polylog}(u))$ in all cases. Note our change of variable in GGV-CSA to make it easier to compare.

\begin{aligned} & \hline Index Space in bits \\ & \hline SAD-CSA [39] $(1+\epsilon) u H_{0}(T)+O(u \log \log \sigma) \\ &$ GGV-CSA [17] $(2+\epsilon) u H_{k}(T)+o(u \log \sigma) \\ &$ AF-FMI [13] $u H_{k}(T)+o(u \log \sigma) \\ &$ Navarro's LZ-index [34] $4 u H_{k}(T)+o(u \log \sigma) \\ &$ ILZI [38] $(5+\epsilon) u H_{k}(T)+o(u \log \sigma) \\ &$ Our LZ-index $(2+\epsilon) u H_{k}(T)+o(u \log \sigma) \\ &$ Our larger LZ-index $(3+\epsilon) u H_{k}(T)+o(u \log \sigma) \\ &$\hline\end{aligned}

\begin{tabular}{|c|c|c|c|}
\hline Index & count & locate & extract \\
\hline SAD-CSA [39] & $O(m \log u)$ & $O\left(\right.$ occ $\left.\log \frac{1}{1+\epsilon} u\right)$ & $O\left(\ell+\log ^{\epsilon} u\right)$ \\
\hline GGV-CSA [17] & $O\left(\frac{m}{\log _{\sigma} u}+\frac{\log \frac{3+\epsilon}{1+\epsilon} u}{\frac{1-\epsilon}{1+\epsilon}}\right)$ & $O\left(\right.$ occ $\left.\log u \log _{\sigma}^{\epsilon} u\right)$ & $O\left(\ell / \log _{\sigma} u+\log u \log _{\sigma}^{\epsilon} u\right)$ \\
\hline AF-FMI [13] & $O(m)$ & $O\left(\right.$ occ $\left.\log ^{1+\epsilon} u\right)$ & $O\left(\ell+\log ^{1+\epsilon} u\right)$ \\
\hline Navarro's LZ-index [34] & $O\left(m^{3} \log \sigma+m \log u+o c c\right)$ & $O($ occ $\log u)$ & $O(\ell \log \sigma)$ \\
\hline ILZI $[38]$ & $O(m \log u+o c c)$ & $O($ occ $\log u)$ & $O\left(\ell / \log _{\sigma} u\right)$ \\
\hline Our LZ-index & $O\left(m^{2}+m \log u+o c c\right)$ & $O($ occ $\log u)$ & $O\left(\ell / \log _{\sigma} u\right)$ \\
\hline Our larger LZ-index & $O(m)$ & $O((m+o c c) \log u)$ & $O\left(\ell / \log _{\sigma} u\right)$ \\
\hline
\end{tabular}

\section{Basic Concepts}

\subsection{Model of Computation}

In this paper we assume the standard word RAM model of computation, in which we can access any memory word of length $w$, such that $w=\Theta(\log u)$, in constant time. Standard arithmetic and 
logical operations (like additions, bit-wise operations, etc.) are assumed to take constant time in this model. We measure the size of our data structures in bits.

\subsection{Empirical Entropy}

A concept related to text compression is that of the $k$-th order empirical entropy of a sequence of symbols $T$ over an alphabet of size $\sigma$, denoted by $H_{k}(T)$ [28]. The value $u H_{k}(T)$ provides a lower bound to the number of bits needed to compress $T$ using any compressor that encodes each symbol considering only the context of $k$ symbols that precede it in $T$. It holds that $0 \leqslant H_{k}(T) \leqslant$ $H_{k-1}(T) \leqslant \cdots \leqslant H_{0}(T) \leqslant \log \sigma$ (log $x$ means $\left\lceil\log _{2} x\right\rceil$ in this paper). Formally, we have

Definition 1. Given a text $T[1 . . u]$ over an alphabet $\Sigma$, the zero-order empirical entropy of $T$ is defined as

$$
H_{0}(T)=\sum_{c \in \Sigma} \frac{n_{c}}{u} \log \frac{u}{n_{c}}
$$

where $n_{c}$ is the number of occurrences of symbol $c$ in $T$. The sum includes only those symbols $c$ that occur in $T$, so that $n_{c}>0$.

Definition 2. Given a text $T[1 . . u]$ over an alphabet $\Sigma$, the $k$-th order empirical entropy of $T$ is defined as

$$
H_{k}(T)=\sum_{s \in \Sigma^{k}} \frac{\left|T^{s}\right|}{u} H_{0}\left(T^{s}\right)
$$

where $T^{s}$ is the subsequence of $T$ formed by all the symbols that occur preceded by the context $s$. Again, we consider only contexts s that do occur in $T$.

\subsection{Ziv-Lempel Compression}

The Ziv-Lempel compression algorithm of 1978 (usually named LZ78 [42]) is based on a dictionary of phrases, in which we add every new phrase computed. At the beginning of the compression, the dictionary contains a single phrase $b_{0}$ of length 0 (i.e., the empty string). The current step of the compression is as follows: If we assume that a prefix $T[1 . . j]$ of $T$ has been already compressed into a sequence of phrases $Z=b_{1} \ldots b_{r}$, all of them in the dictionary, then we look for the longest prefix of the rest of the text $T[j+1 . . u]$ which is a phrase of the dictionary. Once we have found this phrase, say $b_{s}$ of length $\ell_{s}$, we construct a new phrase $b_{r+1}=\left(s, T\left[j+\ell_{s}+1\right]\right)$, write the pair at the end of the compressed file $Z$, i.e. $Z=b_{1} \ldots b_{r} b_{r+1}$, and add the phrase to the dictionary.

We will call $B_{i}$ the string represented by phrase $b_{i}$, thus $B_{r+1}=B_{s} T\left[j+\ell_{s}+1\right]$. In the rest of the paper we assume that the text $T$ has been compressed using the LZ78 algorithm into $n+1$ phrases, $T=B_{0} \ldots B_{n}$, such that $B_{0}=\varepsilon$ (the empty string). We say that $i$ is the phrase identifier corresponding to $B_{i}$, for $0 \leqslant i \leqslant n$.

Property 1. For all $1 \leqslant t \leqslant n$, there exists $\ell<t$ and $c \in \Sigma$ such that $B_{t}=B_{\ell} \cdot c$.

That is, every phrase $B_{t}$ (except $B_{0}$ ) is formed by a previous phrase $B_{\ell}$ plus a symbol $c$ at the end. This implies that the set of phrases is prefix closed, meaning that any prefix of a phrase $B_{t}$ is also an element of the dictionary. Therefore, a natural way to represent the set of strings $B_{0}, \ldots, B_{n}$ is a trie, which we call LZTrie. 
Property 2. Every phrase $B_{i}, 0 \leqslant i<n$, represents a different text substring.

This property is used in the LZ-index search algorithm (see Section 3). The only exception to this property is the last phrase $B_{n}$. We deal with the exception by appending to $T$ a special symbol "\$" $\notin \Sigma$, assumed to be smaller than any other symbol in the alphabet. The last phrase will contain this symbol and thus will be unique too.

In Fig. 1 we show the LZ78 phrase decomposition for our running example text $T=$ "alabar_a_la_alabarda_para_apalabrarla", where for clarity we replace blanks by '_, which is assumed to be lexicographically larger than any other symbol in the alphabet. We show the phrase identifiers above each corresponding phrase in the parsing. In Fig. 5(a) we show the corresponding LZTrie. Inside each LZTrie node we show the corresponding phrase identifier.

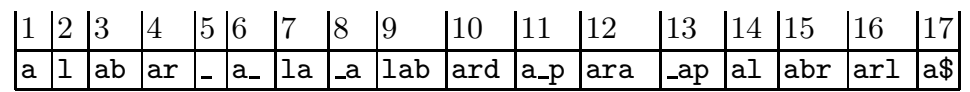

Fig. 1. LZ78 phrase decomposition for the running example text $T$ ="alabar_a_la_alabarda_para_apalabrarla", and the corresponding phrase identifiers.

Definition 3. Let $b_{r}=\left(r_{1}, c_{1}\right), b_{r_{1}}=\left(r_{2}, c_{2}\right), b_{r_{2}}=\left(r_{3}, c_{3}\right)$, and so on until $r_{k}=0$ be phrases of the LZ78 parsing of $T$. The sequence of phrase identifiers $r, r_{1}, r_{2}, \ldots$ is called the referencing chain starting at phrase $r$.

The referencing chain starting at phrase $r$ reproduces the way phrase $b_{r}$ is formed from previous phrases and it is obtained by successively moving to the parent in the LZTrie. For example, the referencing chain of phrase 9 in Fig. 5(a) is $r=9, r_{1}=7, r_{2}=2$, and $r_{3}=0$.

The compression algorithm is $O(u)$ time in the worst case and efficient in practice provided we use the LZTrie, which allows rapid searching of the new text prefix (for each symbol of $T$ we move once in the trie). The decompression needs to build the same dictionary (the pair that defines the phrase $r$ is read at the $r$-th step of the algorithm).

Property 3 ([42]). It holds that $\sqrt{u} \leqslant n \leqslant \frac{u}{\log _{\sigma} u}$. Thus, $\log n=\Theta(\log u)$ and $n \log u \leqslant u \log \sigma$ always hold.

Lemma 1 ([24]). It holds that $n \log n=u H_{k}(T)+O\left(u \frac{1+k \log \sigma}{\log _{\sigma} u}\right)$ for any $k$.

In our work we assume $k=o\left(\log _{\sigma} u\right.$ ) (and hence $\log \sigma=o(\log u)$ to allow for $\left.k>0\right)$; therefore, $n \log n=u H_{k}(T)+o(u \log \sigma)$. Also, in the analysis throughout this paper we will assume $\sigma=$ $O(\operatorname{polylog}(u))$, to finally in Section 8 generalize our results for larger values of $\sigma$.

\subsection{Suffix Arrays and the Burrows-Wheeler Transform}

The suffix array [27] of a text $T[1 . . u]$, denoted by $S A_{T}[1 . . u]$, is a lexicographically sorted array of the text suffixes. Given $i, S A_{T}[i]$ stores the starting text position for the $i$-th suffix in the lexicographic order. A suffix array requires $u \log u$ bits of space if no auxiliary data structure is used [27] and, in such a case, given a pattern $P[1 . . m]$ we are capable to find the suffix-array interval $\left[i_{1}, i_{2}\right]$ containing the starting positions of the pattern occurrences in $O(m \log u)$ time, by binary searching $S A_{T}$ (as 
it is sorted). This corresponds to counting the pattern occurrences, as the size of the interval equals the number of occurrences. To find the starting positions, we traverse the interval $\left[i_{1}, i_{2}\right]$ and report the positions stored in it, in $O(1)$ time per occurrence. In Fig. 2 we show the suffix array for our example text, along with the corresponding (cyclically shifted) text suffix in each case. If we search, for example, for the pattern 'ala', we will get the interval [6..8] in the suffix array (we underline the prefix 'ala' of the corresponding suffixes).

Given a suffix starting at position $S A_{T}[i]$ in $T$, its longest proper suffix has position $S A_{T}[i]+1$ in $T$. Notice that $S A_{T}$ is indeed a permutation, and hence $S A_{T}^{-1}$ denotes its inverse permutation. Given a suffix starting at position $j$ of the text, its lexicographic rank among all suffixes is $S A_{T}^{-1}[j]$.

\begin{tabular}{|c|c|c|c|c|}
\hline$i$ & $S A_{T}[i]$ & suffix (cyclically shifted) & $b t w(T)$ & $\Psi[i]$ \\
\hline 1 & 38 & \$alabar_a_la_alabarda_para_apalabrarla & $\mathrm{a}$ & 7 \\
\hline 2 & 37 & a\$alabar_a_la_alabarda_para_apalabrarl & 1 & 1 \\
\hline 3 & 15 & 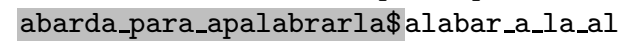 & 1 & 18 \\
\hline 4 & 3 & abar_a_la_alabarda_para_apalabrarla\$al & 1 & 19 \\
\hline 5 & 31 & abrarla\$alabar_a_la_alabarda_para_apal & 1 & 20 \\
\hline 6 & 13 & alabarda_para_apalabrarla\$alabar_a_la_ & _ & 23 \\
\hline 7 & 1 & alabar_a_la_alabarda_para_apalabrarla $\$$ & $\$$ & 24 \\
\hline 8 & 29 & alabrarla\$alabar_a_la_alabarda_para_ap & $\mathrm{p}$ & 25 \\
\hline 9 & 27 & apalabrarla\$alabar_a_la_alabarda_para_ & - & 27 \\
\hline 10 & 23 & ara_apalabrarla\$alabar_a_la_alabarda_p & $\mathrm{p}$ & 30 \\
\hline 11 & 17 & arda_para_apalabrarla\$alabar_a_la_alab & $\mathrm{b}$ & 31 \\
\hline 12 & 34 & arla\$alabar_a_la_alabarda_para_apalabr & $r$ & 32 \\
\hline 13 & 5 & ar_a_la_alabarda_para_apalabrarla $\$ a l a b$ & $\mathrm{~b}$ & 33 \\
\hline 14 & 11 & a_alabarda_para_apalabrarla\$alabar_a_l & 1 & 34 \\
\hline 15 & 25 & a_apalabrarla\$alabar_a_la_alabarda_par & $r$ & 35 \\
\hline 16 & 8 & a_la_alabarda_para_apalabrarla\$alabar_ & - & 37 \\
\hline 17 & 20 & a_para_apalabrarla\$alabar_a_la_alabard & $\mathrm{d}$ & 38 \\
\hline 18 & 16 & barda_para_apalabrarla\$alabar_a_la_ala & $\bar{a}$ & 11 \\
\hline 19 & 4 & bar_a_la_alabarda_para_apalabrarla $\$ a l a$ & a & 13 \\
\hline 20 & 32 & brarla\$alabar_a_la_alabarda_para_apala & $\mathrm{a}$ & 29 \\
\hline 21 & 19 & da_para_apalabrarla\$alabar_a_la_alabar & $r$ & 17 \\
\hline 22 & 36 & la\$alabar_a_la_alabarda_para_apalabrar & $r$ & 2 \\
\hline 23 & 14 & 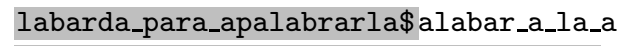 & a & 3 \\
\hline 24 & 2 & labar_a_la_alabarda_para_apalabrarla $\$ a$ & a & 4 \\
\hline 25 & 30 & labrarla\$alabar_a_la_alabarda_para_apa & a & 5 \\
\hline 26 & 10 & la_alabarda_para_apalabrarla\$alabar_a_ & - & 14 \\
\hline$\overline{27}$ & 28 & palabrarla\$alabar_a_la_alabarda_para_a & $\mathrm{a}$ & 8 \\
\hline 28 & 22 & para_apalabrarla\$alabar_a_la_alabarda_ & - & 10 \\
\hline 29 & 33 & rarla\$alabar_a_la_alabarda_para_apalab & $\mathrm{b}$ & 12 \\
\hline 30 & 24 & ra_apalabrarla\$alabar_a_la_alabarda_pa & a & 15 \\
\hline 31 & 18 & rda_para_apalabrarla\$alabar_a_la_alaba & $\mathrm{a}$ & 21 \\
\hline 32 & 35 & rla\$alabar_a_la_alabarda_para_apalabra & a & 22 \\
\hline 33 & 6 & r_a_la_alabarda_para_apalabrarla $\$ a l a b a$ & $\mathrm{a}$ & 36 \\
\hline$\overline{34}$ & 12 & _alabarda_para_apalabrarla\$alabar_a_la & $\mathrm{a}$ & 6 \\
\hline 35 & 26 & _apalabrarla\$alabar_a_la_alabarda_para & a & 9 \\
\hline 36 & 7 & _a_la_alabarda_para_apalabrarla\$alabar & $r$ & 16 \\
\hline 37 & 9 & _la_alabarda_para_apalabrarla\$alabar_a & $\mathrm{a}$ & 26 \\
\hline 38 & 21 & _para_apalabrarla\$alabar_a_la_alabarda & a & 28 \\
\hline
\end{tabular}

Fig. 2. Suffix array for the running example text. Text suffixes are shaded in each row. 
The Burrows-Wheeler Transform [7] of a text $T(b w t(T)$ for short) produces a permutation of the text which is easier to compress than the original text. To compute the transform, after appending the special symbol ' $\$$ ' to $T$, we construct the conceptual matrix $M$ whose rows are the lexicographically-sorted cyclic shifts of $T \$$, and finally take the last column of $M$ as the bwt $(T)$. This is a reversible transform, as we can recover the original text from the bwt $(T)$.

There is a close relation between suffix arrays and the Burrows-Wheeler transform: as every row in $M$ corresponds to a text suffix, and since rows are lexicographically sorted ${ }^{4}$, if we associate to each row the starting position of the suffix what we get is the suffix array of $T$. Also, note that the last column of $M$ (i.e, $b w t(T)$ ) is the symbol preceding each suffix of $T$, that is, $T\left[S A_{T}[i]-1\right]$.

Given this close relation between suffix arrays and the $b w t(T)$, Ferragina and Manzini [12] define the concept of backward search, which consists of looking for the suffix array interval containing the pattern occurrences just using the $b w t(T)$. Given a pattern $P=p_{1} \ldots p_{m}$, for $p_{i} \in \Sigma$, the search proceeds in $O(m)$ steps: in the first step, we find the suffix array interval for the occurrences of $p_{m}$; in the second step, using the result of the previous step, we find the interval corresponding to $p_{m-1} p_{m}$, and so on to finally find the suffix array interval for the whole pattern $p_{1} \ldots p_{m}$. For instance, if we search for the pattern 'bar' in our example of Fig. 2, we first find the interval for the occurrences of ' $r$ ', which is [29..33], then the interval [10..13] for the occurrences of 'ar', to finally find the interval [18..19] corresponding to the occurrences of 'bar'.

The first realization of the backward-search concept was the FM-index [12], yet its use is appropriate only for texts on small alphabets (e.g. constant-size alphabets) because of an exponential dependence on $\sigma$ in the space requirement. A further improvement on this line is the Alphabet-Friendly $F M$-index (AF-FMI) [13], which avoids these alphabet dependences requiring $u H_{k}(T)+o(u \log \sigma)$ bits of space. In this index, each step of the backward search takes $O\left(1+\frac{\log \sigma}{\log \log u}\right)$ time, and thus the counting time is $O\left(m\left(1+\frac{\log \sigma}{\log \log u}\right)\right)$, which is $O(m)$ time whenever $\sigma=$ polylog $(u)$ holds. We are able to locate the pattern occurrences in $O\left(\log ^{1+\epsilon} u \frac{\log \sigma}{\log \log u}\right)$ time per occurrence, provided we store a sampling of the suffix array requiring $o(u \log \sigma)$ extra bits of space. Although requiring "sublinear" space, this extra information we need to store is not compressible at all, as it consists of a sampling of raw suffix-array positions. The time to extract any string of length $\ell$ is $O\left(\left(\ell+\log ^{1+\epsilon} u\right) \frac{\log \sigma}{\log \log u}\right)$.

Another concept related to suffix arrays is that of function $\Psi$ [18], which also allows us to compress the suffix array. This function is defined as $\Psi[i]=S A_{T}^{-1}\left[S A_{T}[i]+1\right]$, for $i=2, \ldots, u$, and $\Psi[1]=S A_{T}^{-1}[1]$. In other words, $\Psi[i]$ stores the suffix-array position (i.e., lexicographic rank) of the longest proper suffix of $S A_{T}[i]$, and hence it can be seen as a suffix link for $S A_{T}[i]$ [41].

A naive way to represent $\Psi$ requires $u \log u$ bits of space, the same as the suffix array itself. However, it can be shown that $\Psi$ can be divided into $\sigma$ strictly increasing sequences: for every $i<j$, if $T\left[S A_{T}[i]\right]=T\left[S A_{T}[j]\right]$ holds, then $\Psi[i]<\Psi[j]$. Thus, $\Psi$ can be represented in the following way in order to require $u H_{0}(T)+o(u \log \sigma)$ bits of space [39]: rather than storing $\Psi[i]$, we store the $\delta$-code [9] of the differences $\Psi[i]-\Psi[i-1]$ if $T\left[S A_{T}[i]\right]=T\left[S A_{T}[j]\right]$. Otherwise we store the $\delta$-code of $\Psi[i]$. In order to access $\Psi[i]$ in constant time, absolute $\Psi$ values are inserted every $\Theta(\log u)$ bits, which adds $O(u)$ bits. To extract an arbitrary position of $\Psi$, we go to the nearest absolute sample before that position and then sequentially advance summing up differences until reaching the desired position. By maintaining a precomputed table with the total number of differences encoded inside every possible chunk of $\frac{\log u}{2}$ bits, we can process each such chunk in constant time,

\footnotetext{
${ }^{4}$ Because of the unique terminator $\$$, sorting the rows is the same as sorting the suffixes.
} 
so the $\Theta(\log u)$ bits of differences can be processed in constant time. The size of that table is only $O\left(\sqrt{u} \log ^{2} u\right)=o(u)$ bits. See [39] for further details.

In Fig. 2 we show $\Psi$ for the running example. We divide the rows of the suffix array to separate suffixes starting with the same symbol. Notice the strictly increasing runs of $\Psi$ within these intervals.

\subsection{Succinct Representations of Sequences and Permutations}

A succinct data structure requires space close to the information-theoretic lower bound, while supporting the corresponding operations efficiently. In this section and the next, we review some results on succinct data structures, which are necessary to understand our work.

Data Structures for rank and select. Given a bit vector $\mathcal{B}[1 . . n]$, we define the operation $\operatorname{rank}_{0}(\mathcal{B}, i)$ (similarly rank $_{1}$ ) as the number of $0 \mathrm{~s}(1 \mathrm{~s})$ occurring up to the $i$-th position of $\mathcal{B}$. The operation $\operatorname{select}_{0}(\mathcal{B}, i)$ (similarly select $t_{1}$ ) is defined as the position of the $i$-th $0(i$-th 1$)$ in $\mathcal{B}$. We assume that $\operatorname{select}_{0}(\mathcal{B}, 0)$ always equals 0 (similarly for select ${ }_{1}$ ). These operations can be supported in constant time and requiring $n+o(n)$ bits [30], or even $n H_{0}(\mathcal{B})+o(n)$ bits [36].

Given a sequence $S[1 . . u]$ over an alphabet $\Sigma$, we generalize the above definition for $\operatorname{rank}_{c}(S, i)$ and $\operatorname{select}_{c}(S, i)$ for any $c \in \Sigma$. If $\sigma=O(\operatorname{polylog}(u))$, the solution of Ferragina at al. [13] allows

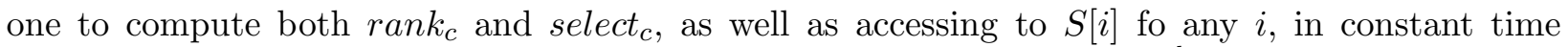
and requiring $u H_{0}(S)+o(u)$ bits of space. Otherwise the time is $O\left(\frac{\log \sigma}{\log \log u}\right)$ and the space is $u H_{0}(S)+o(u \log \sigma)$ bits. The representation of Golynski et al. [16] requires $n(\log \sigma+o(\log \sigma))=$ $O(n \log \sigma)$ bits of space [5], allowing us to compute select $t_{c}$ in $O(1)$ time, and rank $k_{c}$ and access to $S[i]$ in $O(\log \log \sigma)$ time.

Succinct Representation of Permutations. The problem here is to represent a permutation $\pi$ of $\{1, \ldots, n\}$, such that we can compute both $\pi(i)$ and its inverse $\pi^{-1}(j)$ in constant time and using as little space as possible. A natural representation for $\pi$ is to store the values $\pi(i), i=1, \ldots, n$, in an array of $n \log n$ bits. The brute-force solution to the problem computes $\pi^{-1}(j)$ looking for $j$ sequentially in the array representing $\pi$. If $j$ is stored at position $i$, i.e. $\pi(i)=j$, then $\pi^{-1}(j)=i$. Although this solution does not require any extra space to compute $\pi^{-1}$, it takes $O(n)$ time in the worst case.

A much more efficient solution is based on the cycle notation of a permutation. The cycle for the $i$-th element of $\pi$ is formed by elements $i, \pi(i), \pi(\pi(i))$, and so on until the value $i$ is found again. It is important to note that every element occurs in one and only one cycle of $\pi$. For example, the cycle notation for permutation $i d s$ of Fig. 4(a) is shown in Fig. 3. So, to compute $\pi^{-1}(j)$, instead of looking sequentially for $j$ in $\pi$, we only need to look for $j$ in its cycle: $\pi^{-1}(j)$ is just the value "pointing" to $j$ in the diagram of Fig. 3. To compute $i d s^{-1}(13)$ in the previous example, we start at position 13 , then move to position $i d s(13)=7$, then to position $i d s(7)=12$, then to $i d s(12)=2$, then to $i d s(2)=17$, and as $i d s(17)=13$ we conclude that $i d s^{-1}(13)=17$. The only problem here is that there are no bounds for the size of a cycle, hence this algorithm takes also $O(n)$ in the worst case. However, it can be improved for a more efficient computation of $\pi^{-1}(j)$.

Given $0<\epsilon<1$, we create subcycles of size $O(1 / \epsilon)$ by adding a backward pointer out of $O(1 / \epsilon)$ elements in each cycle of $\pi$. Dashed arrows in Fig. 3 show backward pointers for $1 / \epsilon=2$. To compute $i d s^{-1}(17)$, we first move to $i d s(17)=13$; as 13 has a backward pointer we follow it and hence we move to position 2 . Then, as $i d s(2)=17$ we conclude that $i d s^{-1}(17)=2$, in $O(1 / \epsilon)$ 

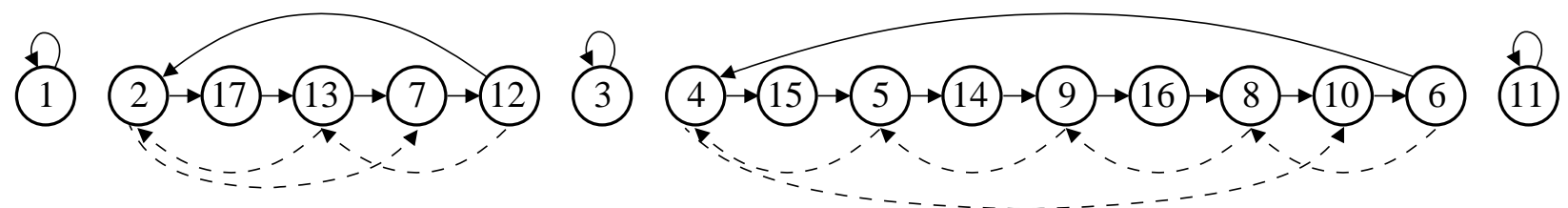

Fig. 3. Cycle representation of permutation $i d s$ of Fig. 4(a). Each solid arrow $i \rightarrow j$ in the diagram means $i d s(i)=j$. Dashed arrows represent backward pointers.

worst-case time. We store the backward pointers compactly in an array of $\epsilon n \log n$ bits. We mark the elements having a backward pointer by using a bit vector supporting rank queries, which also help us to find the backward pointer corresponding to a given element (see [31] for details). Overall, this solution requires $(1+\epsilon) n \log n+n+o(n)$ bits of storage.

\subsection{Succinct Representation of Trees}

Given a tree with $n$ nodes, there exist a number of succinct representations requiring $2 n+o(n)$ bits, which is close to the information-theoretic lower bound of $2 n-\Theta(\log n)$ bits. We explain the representations that we will need in our work.

Balanced Parentheses. The balanced parentheses representation of Munro and Raman [32] is built from a depth-first preorder traversal of the tree, writing an opening parenthesis when arriving to a node for the first time, and a closing parenthesis when going up (after traversing the subtree of the node). In this way, each node is represented by a pair of opening and closing parentheses. We identify a tree node $x$ with its opening parenthesis in the representation. The subtree of $x$ contains those nodes (parentheses) enclosed between the opening parenthesis representing $x$ and its matching closing parenthesis.

The preorder position of a node can be computed in this representation as the number of opening parentheses before the one representing the node. That is, $\operatorname{preorder}(x) \equiv \operatorname{rank}_{(}(\operatorname{par}, i)-1$, where par represents the balanced parentheses sequence over the alphabet $\{()$,$\} . Notice that in this way$ the preorder of the tree root is always 0 . Given a preorder position $p$, the corresponding node is computed by select ( $(\mathrm{par}, p)$, assuming that the parentheses sequence starts at position 0 .

This representation requires $2 n+o(n)$ bits, allowing operations parent $(x)$ (which gets the parent of node $x$ ), subtreesize $(x)$ (which gets the size of the subtree of node $x$, including $x$ itself), depth $(x)$ (which gets the depth of node $x$ in the tree), and ancestor $(x, y)$ (which tell us whether node $x$ is an ancestor of node $y$ ), all of them in $O(1)$ time. Operation child $(x, i)$ (which gets the $i$-th child of node $x$ ) can be computed in $O(i)$ time.

In Fig. 4(a) we show the balanced parentheses representation for the LZTrie of Fig. 5(a), along with the sequence of phrase identifiers $(i d s)$ in preorder, and the sequence of symbols labeling the edges of the trie (letts), also in preorder. As the identifier corresponding to the LZTrie root is always 0 , we do not store it in $i d s$. The data associated with node $x$ is stored at position preorder $(x)$ both in $i d s$ and letts sequences. Note this information is sufficient to reconstruct LZTrie.

DFUDS Representation. To get this representation [6] we perform a preorder traversal on the tree, and for every node reached we write its degree in unary using parentheses. For example, 3 reads 


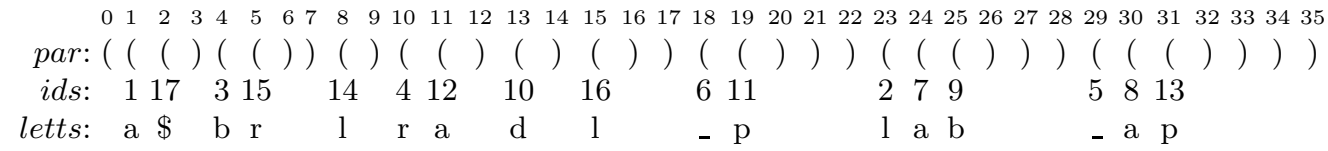

(a) Balanced parentheses representation of LZTrie for the running example.

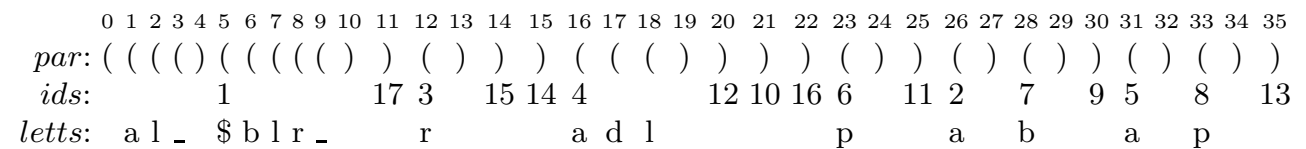

(b) DFUDS representation of LZTrie for the running example. The phrase identifiers are stored in preorder, and the symbols labeling the edges of the trie are stored according to DFUDS.

Fig. 4. Succinct representations of LZTrie for the running example.

' $((()$ ' under this representation. What we get is almost a balanced parentheses representation: we only need to add a fictitious ' (' at the beginning of the sequence. A node of degree $d$ is identified by the position of the first of the $(d+1)$ parentheses representing the node. Given a node $x$ in this representation, say at position $i$, its preorder position can be computed by counting the number of closing parentheses before position $i$; in other words, $\operatorname{preorder}(x) \equiv \operatorname{rank}(\operatorname{par}, i-1)$, where par represents the DFUDS sequence of the tree. Given a preorder position $p$, the corresponding node is computed by select ( par, $p)+1$.

This representation requires also $2 n+o(n)$ bits, and we can compute operations parent $(x)$, subtreesize $(x)$, degree $(x)$ (which gets the degree, i.e. the number of children, of node $x$ ), ancestor $(x$, $y),{ }^{5}$ and $\operatorname{child}(x, i)$, all in $O(1)$ time. Operation $\operatorname{depth}(x)$ can also be computed in constant time on DFUDS by using the approach of Jansson, Sadakane, and Sung [21], requiring o( $n)$ extra bits.

For cardinal trees (i.e., trees where each node has at most $\sigma$ children, each child labeled by a symbol in the set $\{1, \ldots, \sigma\})$ we use the DFUDS sequence par plus an array letts[1..n] storing the edge labels according to a DFUDS traversal of the tree: we traverse the tree in depth-first preorder, and every time we reach a node $x$ we write the symbols labeling the children of $x$. In this way, the labels of the children of a given node are all stored contiguously in letts, which will allow us to compute operation $\operatorname{child}(x, \alpha)$ (which gets the child of node $x$ with label $\alpha \in\{1, \ldots, \sigma\}$ ) efficiently. In Fig. 4(b) we show the DFUDS representation of LZTrie for our running example.

We solve operation $\operatorname{child}(x, \alpha)$ as follows. Suppose that node $x$ has position $p$ within the DFUDS sequence $p a r$, and let $p^{\prime}=\operatorname{rank}_{(}($par, $p)-1$ be the position in letts for the symbol of the first child of $x$. Let $n_{\alpha}=\operatorname{rank}_{\alpha}\left(\right.$ letts, $\left.p^{\prime}-1\right)$ be the number of $\alpha$ s up to position $p^{\prime}-1$ in letts, and let $i=$ select $_{\alpha}\left(\right.$ letts, $\left.n_{\alpha}+1\right)$ be the position of the $\left(n_{\alpha}+1\right)$-th $\alpha$ in letts. If $i$ lies within positions $p^{\prime}$ and $p^{\prime}+\operatorname{degree}(x)-1$, then the child we are looking for is $\operatorname{child}\left(x, i-p^{\prime}+1\right)$, which, as we said before, is computed in constant time over par; otherwise $x$ has not a child labeled $\alpha$. We can also retrieve the symbol by which $x$ descends from its parent with letts $\left[\operatorname{rank}_{(}(\right.$par, parent $\left.(x))-1+\operatorname{childrank}(x)-1\right]$, where the first term stands for the position in letts corresponding to the first symbol of the parent of node $x$, and the second term $\operatorname{childrank}(x)$ is the rank of node $x$ within its siblings, which can be computed in constant time [21].

Thus, the time for operation $\operatorname{child}(x, \alpha)$ depends on the representation we use for $\operatorname{rank}_{\alpha}$ and select $_{\alpha}$ queries. Notice that $\operatorname{child}(x, \alpha)$ could be solved in a straightforward way by binary searching

\footnotetext{
${ }^{5}$ As ancestor $(x, y) \equiv \operatorname{preorder}(x) \leqslant \operatorname{preorder}(y) \leqslant \operatorname{preorder}(x)+$ subtreesize $($ par,$x)-1$.
} 
the labels of the children of $x$, in $O(\log \sigma)$ worst-case time and not needing any extra space on top of array letts. The access to letts[.] takes constant time.

We can represent letts with the data structure of Ferragina et al. [13], which requires $n \log \sigma+$ $o(n \log \sigma)$ bits of space, and allows us to compute $\operatorname{child}(x, \alpha)$ in $O\left(\frac{\log \sigma}{\log \log u}\right)$ time. The access to letts [.] also takes $O\left(\frac{\log \sigma}{\log \log u}\right)$ time. These times are $O(1)$ whenever $\sigma=O(\operatorname{polylog}(u))$ holds. On the other hand, we can use the data structure of Golynski et al. [16], requiring $O(n \log \sigma)$ bits of space, yet allowing us to compute $\operatorname{child}(x, \alpha)$ in $O(\log \log \sigma)$ time, and access to letts[.] also in $O(\log \log \sigma)$ time. In most of this paper we will use the representation of Ferragina et al. to represent the symbols, since it is able to improve its time complexity to $O(1)$ for smaller alphabets.

The scheme we have presented to represent letts is slightly different to the original one [6], which achieves $O(1)$ time for $\operatorname{child}(x, \alpha)$ for any $\sigma$. However, ours is simpler and allows us to efficiently access letts[·], which will be very important in our indices to extract text substrings. We need to store the array of symbols explicitly in case we need to access them (fortunately, this will not asymptotically affect the space requirement of our results).

$\boldsymbol{x} \boldsymbol{b} \boldsymbol{w}$ Representation. The $x b w$ transform of Ferragina et al. [11] is a succinct representation for labeled trees: Given a labeled tree $\mathcal{T}$, with $n$ nodes and labels taken from an alphabet $\Sigma$ of size $\sigma$, the $x b w$ transform of $\mathcal{T}$ is computed by first traversing the tree in preorder, and for each node writing a triplet in a table $S_{\mathcal{T}}$. The first component of each triplet indicates whether the node is the last child of its parent in the tree, the second component is the symbol labeling the edge by which we reach the node, and the third component is the string labeling the path from the parent of the node to the root of $\mathcal{T}$. In this way each node is represented by a row in $S_{\mathcal{T}}$. As in the original work [11], we call $S_{\text {last }}, S_{\alpha}$, and $S_{\pi}$ the columns of table $S_{\mathcal{T}}$, storing respectively the first, second and third components of each triplet. As a last step we perform an upward-path-sorting of the table by stably sorting the rows of $S_{\mathcal{T}}$ lexicographically according to the strings in $S_{\pi}$.

In Table 2 we show the $x b w$ transform for the LZTrie of Fig. 5(a). We have to add a dummy child to each leaf, labeling the dummy edge with a special symbol $\Delta$ not in $\Sigma$, so that the paths leading to the leaves appear in column $S_{\pi}$, and hence later we will be able of searching for them. As we said before, each node in the LZTrie is represented by a row in the table, being the row number what we call the $x b w$ position of the node.

The $x b w$ representation supports operations parent $(x)$, $\operatorname{child}(x, i)$, and $\operatorname{child}(x, \alpha)$, all of them in $O(1)$ time if $\sigma=O($ polylog $(u))$, and using $2 n \log \sigma+O(n)$ bits of space, because the column $S_{\pi}$ of the table is not stored. The representation also allows subpath queries, a very powerful operation which, given a string $s$, returns all the nodes $x$ such that $s$ is a prefix of the string labeling the path from the parent of $x$ to the root. If $\sigma=O(\operatorname{polylog}(n))$, subpath queries can be computed in $O(|s|)$ time [11]. For general $\sigma$, the time for all these operations depends on the representation used for $S_{\alpha}$ (since we need to support rank and select operations on it), which is $O\left(\frac{\log \sigma}{\log \log u}\right)$ time if we use the representation of [13], and $O(\log \log \sigma)$ time if we use the data structure of [16], in which case the space requirement is $O(n \log \sigma)$.

Because of the upward-path sorting in table $S_{\mathcal{T}}$, the result of a subpath query is a contiguous interval in such table, containing the answers to the query. For example, a subpath query for string ' $r$ ' yields the interval [21..24] in Table 2, corresponding respectively to the nodes with preorders 7, 8, and 9 in Fig. 5(a), plus a fictitious leaf which is a child of node with preorder 4. As another example, a subpath query for string 'ba' yields the $x b w$ interval [13..14], for node with preorder 4 
plus a fictitious leaf which is a child of node with preorder 14 . In all cases, note that the string $s$ we are looking for is a prefix of the corresponding string in $S_{\pi}$.

Table 2. $x b w$ representation for the LZTrie of Fig. 5(a).

\begin{tabular}{cccl}
\hline$i$ & $S_{\text {last }}$ & $S_{\alpha}$ & $S_{\pi}$ \\
\hline 1 & 0 & a & empty string \\
2 & 0 & 1 & empty string \\
3 & 1 & - & empty string \\
4 & 1 & $\Delta$ & $\$$ a \\
5 & 0 & $\$$ & a \\
6 & 0 & b & a \\
7 & 0 & l & a \\
8 & 0 & r & a \\
9 & 1 & - & a \\
10 & 1 & b & al \\
11 & 1 & $\Delta$ & ara \\
12 & 1 & p & a_ \\
13 & 1 & r & ba \\
14 & 1 & $\Delta$ & bal \\
15 & 1 & $\Delta$ & dra \\
16 & 1 & a & 1 \\
17 & 1 & $\Delta$ & la \\
18 & 1 & $\Delta$ & lra \\
19 & 1 & $\Delta$ & pa_ \\
20 & 1 & $\Delta$ & p_a \\
21 & 0 & a & ra \\
22 & 0 & d & ra \\
23 & 1 & l & ra \\
24 & 1 & $\Delta$ & rba \\
25 & 1 & a & - \\
26 & 1 & p & -a \\
\hline
\end{tabular}

\section{The LZ-index Data Structure}

Assume that the text $T[1 . . u]$ has been compressed using the LZ78 algorithm into $n+1$ phrases $T=B_{0} \ldots B_{n}$, as explained in Section 2.3. Next we describe the original LZ-index data structure and search algorithms, and introduce some first improvements on them.

Hereafter, given a string $S=s_{1} \ldots s_{i}$, we will use $S^{r}=s_{i} \ldots s_{1}$ to denote its reverse. Moreover, $S^{r}[i . . j]$ will actually mean $(S[i . . j])^{r}$.

\subsection{Original LZ-index Components}

The following data structures conform the original LZ-index [34,33]:

1. LZTrie: is the trie formed by all the phrases $B_{0} \ldots B_{n}$. Given the properties of LZ78 compression, this trie has exactly $n+1$ nodes, each one corresponding to a string.

2. RevTrie: is the trie formed by all the reverse strings $B_{0}^{r} \ldots B_{n}^{r}$. In this trie there could be internal nodes not representing any phrase. We call these nodes "empty". 
3. Node: is a mapping from phrase identifiers to their node in LZTrie.

4. Range: is a data structure for two-dimensional searching in the space $[0 . . n] \times[0 . . n]$. We store the points $\{($ revpreorder $(t)$, preorder $(t+1)), t \in 0 \ldots n-1\}$ in this structure, where revpreorder $(t)$ is the RevTrie preorder of the node for phrase $t$ (considering only the non-empty nodes in the preorder enumeration), and preorder $(t+1)$ is the LZTrie preorder of node for phrase $t+1$. For each such point, the corresponding $t$ value is stored.

Fig. 5 shows the LZTrie, RevTrie, Range, and Node data structures corresponding to our running example. We show preorder numbers, both in LZTrie and RevTrie (in the latter case only counting non-empty nodes), outside each trie node. In the case of RevTrie, empty nodes are shown in light gray. The next example gives a hint on the usage of those structures for searching, which will be detailed in Section 3.3.

Example 1. To find all phrases ending with substring 'ab' in the running example, we search for the reversed string 'ba' in RevTrie, reaching the node with preorder 6 . The subtree of this RevTrie node contains the phrases we are looking for: phrases 3 and 9 (see Fig. 1). As the preorder interval in RevTrie defined by this subtree is [6..7], this means that the horizontal semi-infinite range $[-\infty . . \infty] \times[6 . .7]$ in Range also contains those phrases. To find all phrases starting with 'ar', note that the LZTrie subtree for node with preorder (incidentally also) 6 (which corresponds to string 'ar') contains the phrases starting with 'ar': phrases 4, 12, 10, and 16. The LZTrie preorder interval for this subtree is [6..9]. This means that the vertical semi-infinite range $[6 . .9] \times[-\infty . . \infty]$ contains phrases $i$ such that phrase $i+1$ starts with 'ar': phrases 3, 11, 9, and 15. Finally, the range $[6 . .9] \times[6 . .7]$ contains the phrase numbers $i$ such that phrase $i$ ends with 'ab' followed by phrase $i+1$ starting with 'ar': phrases 3 and 9, see Fig. 5(c).

\subsection{Succinct Representation of the LZ-index Components}

In the original work [34], each of the four structures described requires $n \log n+o(u \log \sigma)$ bits of space if they are represented succinctly.

- LZTrie is represented using the balanced parentheses representation [32] requiring $2 n+o(n)$ bits; plus the sequence letts of symbols labeling each trie edge, requiring $n \log \sigma$ bits; and the sequence $i d s$ of $n \log n$ bits storing the LZ78 phrase identifiers. Both letts and $i d s$ are stored in preorder, so we use preorder $(x)$ to index them. See Fig. 4(a) for an illustration.

- For RevTrie, balanced parentheses are also used to represent the Patricia tree [29] structure of the trie, compressing empty unary nodes and so ensuring $n^{\prime} \leqslant 2 n$ nodes. This requires $4 n+o(n)$ bits. The RevTrie-preorder sequence of identifiers (rids) is stored in $n \log n$ bits. The symbols labeling the edges of the trie and the Patricia-tree skips are not stored in this representation, since they can be retrieved by using the connection with LZTrie [34]. Therefore, the navigation on RevTrie is more expensive than that on LZTrie.

- For Range, the data structure of Chazelle [8] permits two-dimensional range searching in a grid of $n$ pairs of integers in the range $[0 . . n] \times[0 . . n]$, answering queries in $O((o c c+1) \log n)$ time, where occ is the number of occurrences reported, and requiring $n \log n+O(n \log \log n)$ bits of space [25]; note that the last term $O(n \log \log n)$ is $o(u \log \sigma)$. This data structure can count the number of points in a given range in $O(\log n)$ time. 


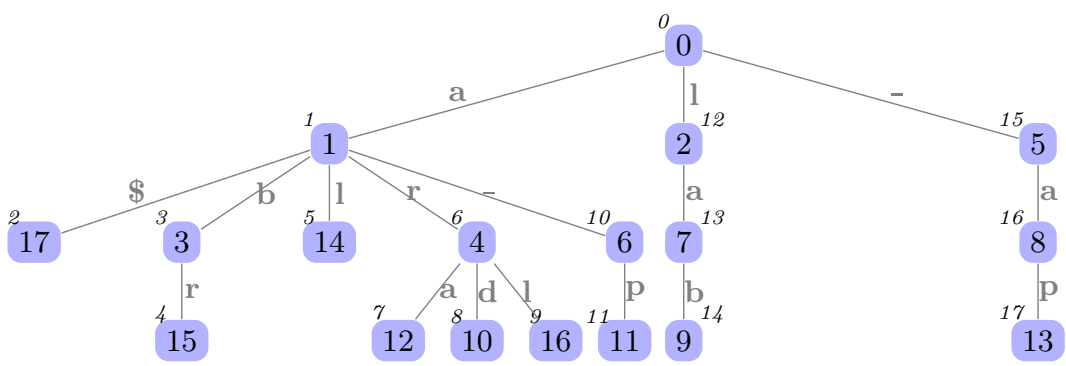

(a) Lempel-Ziv Trie (LZTrie) for the running example.

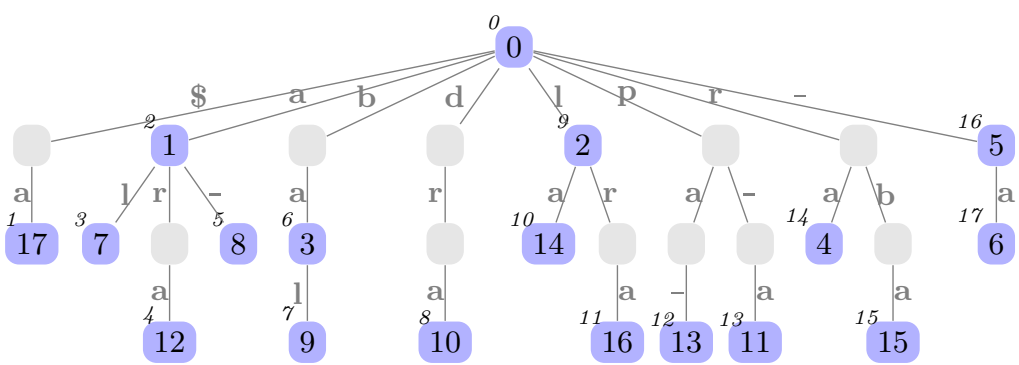

(b) RevTrie data structure.

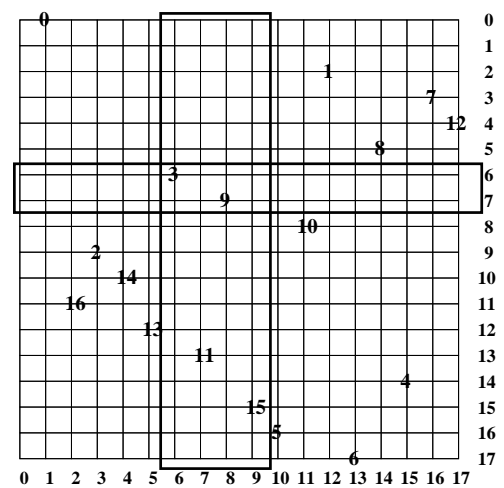

(c) Range data structure. Horizontal coordinates are for LZTrie preorders, and vertical coordinates are for RevTrie preorders.

\begin{tabular}{rlllllllllllllllllll}
\hline$i:$ & 0 & 1 & 2 & 3 & 4 & 5 & 6 & 7 & 8 & 9 & 10 & 11 & 12 & 13 & 14 & 15 & 16 & 17 \\
\hline Node[i] : & 0 & 1 & 23 & 4 & 10 & 29 & 18 & 24 & 30 & 25 & 13 & 19 & 11 & 31 & 8 & 5 & 15 & 2
\end{tabular}

(d) Node data structure, assuming that the parentheses sequence starts from position zero, cf. Fig. 4(a).

Fig. 5. LZ-index components for the running example. 
- Finally, Node is just a sequence of $n$ pointers to LZTrie nodes. As LZTrie is implemented using balanced parentheses, Node $[i]$ stores the position within the sequence for the opening parenthesis representing the node corresponding to phrase $i$. As there are $2 n$ such positions, we need $n \log 2 n=n \log n+n$ bits of storage. See Fig. $5(\mathrm{~d})$ for an illustration.

According to Lemma 1, the final size of the LZ-index is $4 u H_{k}(T)+o(u \log \sigma)$ bits for $k=o\left(\log _{\sigma} u\right)$ (and hence $\log \sigma=o(\log u$ ) if we want to allow for $k>0$ ).

The succinct trie representations used in [34] implement (among others) operations parent $(x)$ and $\operatorname{child}(x, \alpha)$, both in $O(\log \sigma)$ time for LZTrie, and $O(\log \sigma)$ and $O(h \log \sigma)$ time respectively for RevTrie, where $h$ is the depth of node $x$ in RevTrie (the $h$ in the cost comes from the fact that we must access LZTrie to get the label of a RevTrie edge). The operation ancestor $(x, y)$ is implemented in $O(1)$ time both in LZTrie and RevTrie.

\subsection{LZ-index Search Algorithm}

Let us consider now the search algorithm for a pattern $P[1 . . m][33,34]$. For locate queries, pattern

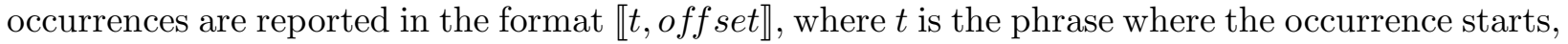
and off set is the distance between the beginning of the occurrence and the end of the phrase. Later, in Section 7, we will show how to map these two values into a single text position. As we deal with an implicit representation of the text (the LZTrie), and not the text itself, we distinguish three types of occurrences of $P$ in $T$, depending on the phrase layout.

Occurrences of Type 1. The occurrence lies inside a single phrase (there are occ $_{1}$ occurrences of this type). Given the properties of LZ78, every phrase $B_{t}$ containing $P$ is formed by a shorter phrase $B_{\ell}$ concatenated to a symbol $c$ (Property 1). If $P$ does not occur at the end of $B_{t}$, then $B_{\ell}$ contains $P$ as well. We want to find the shortest possible phrase $B_{i}$ in the LZ78 referencing chain for $B_{t}$ that contains the occurrence of $P$. Since we can only perform prefix searching with LZTrie, and since phrase $B_{i}$ has the string $P$ as a suffix, we cannot use the LZTrie to find $B_{i}$. But note that $P^{r}$ is a prefix of $B_{i}^{r}$, therefore it can be easily found by searching for $P^{r}$ in RevTrie in $O\left(m^{2} \log \sigma\right)$ time. Say we arrive at node $v_{r}$. Any node $v_{r}^{\prime}$ descending from $v_{r}$ in RevTrie (including $v_{r}$ itself) corresponds to a phrase terminated with $P$. Notice the relation with subpath queries (see Section 2.5). For each such $v_{r}^{\prime}$, we traverse and report the subtree of the corresponding LZTrie node $v_{l z}$ (found using rids and Node). For any node $v_{l z}^{\prime}$ in the subtree of $v_{l z}$, we report an occurrence $\llbracket t, m+\left(\operatorname{depth}\left(v_{l z}^{\prime}\right)-\operatorname{depth}\left(v_{l z}\right)\right) \rrbracket$, where $t$ is the phrase identifier $(i d s)$ of node $v_{l z}^{\prime}$. Occurrences of type 1 are located in $O\left(m^{2} \log \sigma+o c c_{1}\right)$ time, since each occurrence takes constant time in LZTrie.

Occurrences of Type 2. The occurrence spans two consecutive phrases, $B_{t}$ and $B_{t+1}$, such that a prefix $P[1 . . i]$ matches a suffix of $B_{t}$ and the suffix $P[i+1 . . m]$ matches a prefix of $B_{t+1}$ (there are $o c c_{2}$ occurrences of this type). $P$ can be split at any position, so we have to try them all. The idea is that, for every possible split, we search for the reverse pattern prefix $P^{r}[1 . . i]$ in RevTrie (getting node $v_{r}$ ) and for the pattern suffix $P[i+1 \ldots m]$ in LZTrie (getting node $v_{l z}$ ). As in a trie all the strings represented in a subtree form a preorder interval, we have two preorder intervals: One in the space of reversed phrases (phrases finishing with $P[1 . . i]$ ) and one in that of the normal phrases (phrases starting with $P[i+1 . . m]$ ), and need to find the phrase pairs $(t, t+1)$ such that $t$ is in the RevTrie preorder interval and $t+1$ is in the LZTrie preorder interval. As we have seen in Example 1, this is what the range searching data structure (Range) is for: we search Range for 
$\left[\right.$ preorder $\left(v_{l z}\right)$. .preorder $\left(v_{l z}\right)+$ subtreesize $\left.\left(v_{l z}\right)-1\right] \times\left[\operatorname{preorder}\left(v_{r}\right)\right.$. .preorder $\left(v_{r}\right)+\operatorname{subtreesize}\left(v_{r}\right)-$ $1]$. For every pair $(t, t+1)$ found we report occurrence $\llbracket t, i \rrbracket$. Occurrences of type 2 are located in $O\left(m^{3} \log \sigma+\left(m+o c c_{2}\right) \log n\right)$ time, where the first term comes from searching the tries (in particular, searching for the $O(m)$ partitions of $P$ in the RevTrie), and the second one is for the $m-1$ range searches on RevTrie.

Occurrences of Type 3. The occurrence spans three or more phrases, $B_{t-1}, \ldots, B_{\ell+1}$, such that $P[i . . j]=B_{t} \ldots B_{\ell}, P[1 . . i-1]$ matches a suffix of $B_{t-1}$ and $P[j+1 . . m]$ matches a prefix of $B_{\ell+1}$ (there are occ $_{3}$ occurrences of this type). We need one more observation for this part: Since the LZ78 algorithm guarantees that every phrase represents a different string (Property 2), there is at most one phrase matching $P[i . . j]$ for each choice of $i$ and $j$. Therefore, if we partition $P$ into more than two consecutive substrings, there is at most one pattern occurrence for such partition, which severely limits $\mathrm{occ}_{3}$ to $O\left(\mathrm{~m}^{2}\right)$, since this is the number of different partitions of $P$.

Let us define matrix $C_{l z}[1 . . m, 1 . . m]$ and arrays $A_{i}$, for $1 \leqslant i \leqslant m$, which store information about the search. We first identify the only possible phrase matching each substring $P[i . . j]$. This is done by searching for every pattern substring $P[i . . j]$ in LZTrie, for increasing $i$ and for each $i$ value we increase $j$. Thus, we perform a single search in the trie for each $i$. We record in $C_{l z}[i, j]$ the LZTrie node corresponding to $P[i . . j]$, and store the pair $(i d, j)$ at the end of $A_{i}$, such that $i d$ is the phrase identifier of the node corresponding to $P[i . . j]$. Note that since we search for $P[i . . j]$ for increasing $j$, we get the values of $i d$ in increasing order, as the phrase identifier of a node is always larger than that of the parent node. Therefore, the corresponding pairs in $A_{i}$ are stored by increasing value of $i d$. This process takes $O\left(m^{2} \log \sigma\right)$ time.

Then we find the $O\left(\mathrm{~m}^{2}\right)$ maximal concatenations of successive phrases that match contiguous pattern substrings. For $1 \leqslant i \leqslant j \leqslant m$, for increasing $j$, we try to extend the match of $P[i . . j]$ to the right. If $i d$ is the phrase identifier for node $C_{l z}[i, j]$, then we have to search for $(i d+1, r)$ in array $A_{j+1}$, for some $r$. Array $A_{j+1}$ can be binary searched because it is sorted. If we find $(i d+1, r)$ in $A_{j+1}$, this means that $B_{i d}=P[i . . j]$ and $B_{i d+1}=P[j+1 . . r]$, which also means that the concatenation of phrases $B_{i d} B_{i d+1}$ equals $P[i . . r]$. We repeat the process from $j=r$, and stop when the pair $(i d+1, r)$ is not found in the corresponding array (this means that a concatenation of phrases cannot be extended further, so the current concatenation is maximal). See [34] for further details. As we have to perform $O\left(\mathrm{~m}^{2}\right)$ binary searches in arrays of size $O(m)$, this procedure takes $O\left(m^{2} \log m\right)$ worst-case time. In practice, the binary search is replaced by hashing schemes, taking $O\left(m^{2}\right)$ time on average [34, Section 6.5].

If $P[i . . j]=B_{t} \ldots B_{\ell}$ is a maximal concatenation, then we check whether phrase $B_{\ell+1}$ starts with $P[j+1 . . m]$, that is, we check whether $N o d e[\ell+1]$ is a descendant of node $C_{l z}[j+1, m]$, in constant time per maximal concatenation. Finally we check whether phrase $B_{t-1}$ ends with $P[1 . . i-1]$, by starting from Node $[i-1]$ in LZTrie and successively going to the parent to check whether the last $i-1$ symbols, read upwards, equal $P^{r}[1 . . i-1]$, in $O(m \log \sigma)$ time per maximal concatenation. If all these conditions hold, we report an occurrence $\llbracket t-1, i-1 \rrbracket$. Overall, occurrences of type 3 are located in $O\left(m^{3} \log \sigma\right)$ time.

Overall Query Time. Note that each of the occ $=o c c_{1}+o c c_{2}+o c c_{3}$ possible occurrences of $P$ lies exactly in one of the three cases above. Overall, the total search time to report the occ occurrences of $P$ in $T$ is $O\left(m^{3} \log \sigma+(m+o c c) \log u\right)$. 
Extracting Text Substrings. The original LZ-index is able to extract text substrings, yet not in the way we have defined before: we have to provide an LZ78 phrase number from where to start the extraction. We assume also that the $\ell$ symbols we want to extract correspond to whole phrases (in Section 7 we shall avoid all of this restrictions). Given phrase $i$, we follow the upward path from Node $[i]$ up to the LZTrie root, outputting the symbols labeling the upward path. Then we perform the same procedure but now starting from Node $[i+1]$ in LZTrie, and so on until we extract the $\ell$ desired symbols, taking overall $O(\ell \log \sigma)$ time, because operation parent is implemented in $O(\log \sigma)$ time [34]. Finally, we can uncompress the whole text $T$ in $O(u \log \sigma)$ time using the same idea, starting the procedure from the first LZ78 phrase.

Improving the Algorithm for Finding Maximal Concatenations. In the case of occurrences of type 3, we now improve the algorithm for finding maximal concatenation of phrases, replacing the binary searches on arrays $A_{i}$ by an access to the correct position in matrix $C_{l z}$.

When computing maximal concatenations of phrases, for each $1 \leqslant i \leqslant j \leqslant m$, for increasing $j$, we try to extend the match of $P[i . . j]$ to the right. Let $i d$ be the phrase identifier for node $C_{l z}[i, j]$, then $P[i . . j]=B_{i d}$ holds. Then, to check whether $P[j+1 . . r]=B_{i d+1}$ holds, instead of searching for $(i d+1, r)$ in $A_{j+1}$ as before, we note that $r=j+l$, where $l$ is the length of phrase $B_{i d+1}$, which in turn is computed as $l=\operatorname{depth}(N o d e[i d+1])$ in LZTrie. Then we check, in constant time, whether the node $C_{l z}[j+1, j+l]$ corresponds to identifier $i d+1$. This means that $P[j+1 . . r]=B_{i d+1}$ holds, for $r=j+l$, and hence we can extend the concatenation of phrases to $P[i . r]=B_{i d} B_{i d+1}$. We repeat the process for $j=r$ and stop the procedure when the above condition does not hold, or $r$ becomes greater than $m$.

Note that by using this algorithm to find maximal concatenations we reduce the time to $O\left(\mathrm{~m}^{2}\right)$, which does not improve the total performance of the algorithm for finding occurrences of type 3. However, the reduction will be relevant in Sections 4 and 5 for improved versions of the LZ-index.

Lemma 2. Given the $L Z 78$ parsing of text $T \$=B_{0} \ldots B_{n}$, and given a pattern $P[1 . . m]$, we can compute the maximal concatenation of successive phrases $B_{t} \ldots B_{\ell}$ that match contiguous pattern substrings $P[i . . j]$, for any $0 \leqslant i \leqslant j \leqslant m$, in $O\left(m^{2}\right)$ time overall.

\subsection{A Reduced Version of the LZ-index}

In the practical implementation of LZ-index [34,33], the Range data structure defined in Section 3.1 is replaced by $R N o d e$, which is a mapping from phrase identifiers to their node in RevTrie. The $R N o d e$ data structure requires $n \log n$ bits, and so this practical version of LZ-index also requires $4 u H_{k}(T)+o(u \log \sigma)$ bits, for any $k=o\left(\log _{\sigma} u\right)$.

Now occurrences of type 2 are found as follows: For every possible split $P[1 . . i]$ and $P[i+1 . . m]$ of $P$, assume the search for $P^{r}[1 . . i]$ in RevTrie yields node $v_{r}$, and the search for $P[i+1 . . m]$ in LZTrie yields node $v_{l z}$. Then, one checks each phrase $t$ in the subtree of $v_{r}$ and reports it if Node $[t+1]$ descends from $v_{l z}$. Each such check takes constant time. Yet, if the subtree of $v_{l z}$ has fewer elements, one does the opposite: check phrases from $v_{l z}$ in $v_{r}$, using $R N o d e[t-1]$. Unlike when using Range, now the time to solve occurrences of type 2 is proportional to the smallest subtree size among $v_{r}$ and $v_{l z}$, which can be arbitrarily larger than the number of occurrences reported. That is, by using RNode we have no worst-case guarantees at search time. However, the average search time for occurrences of type 2 is $O\left(n / \sigma^{m / 2}\right)[34,33]$. This is $O(1)$ for long patterns, $m \geqslant 2 \log _{\sigma} n$. 
For occurrences of type 3 , after finding that $P[i . . j]=B_{t} \ldots B_{\ell}$ is a maximal concatenation, one checks whether phrase $B_{\ell+1}$ starts with $P[j+1 . . m]$ by using operation ancestor $\left(C_{l z}[j+\right.$ $1, m]$, Node $[\ell+1])$, just as in Section 3.3. To check whether phrase $B_{t-1}$ ends with $P[1 . . i-1]$, instead of performing a symbol-per-symbol checking in LZTrie, as done in the original LZ-index, one simply checks whether ancestor $\left(C_{l z}[1, i-1], R N o d e[t-1]\right)$ holds in RevTrie.

This version of the LZ-index can be seen as a navigation scheme, as shown in Fig. 6, where solid arrows represent the main data structures of the index. Dashed arrows are asymptotically "for free" in terms of space requirement, since they are followed by applying rank on the corresponding parentheses structure (see Section 2.5). The four solid arrows are in fact the four main components in the space usage of the index: array of phrase identifiers in LZTrie (ids) and in RevTrie (rids), and mapping from phrase identifiers to tree nodes in LZTrie (Node) and in RevTrie (RNode).

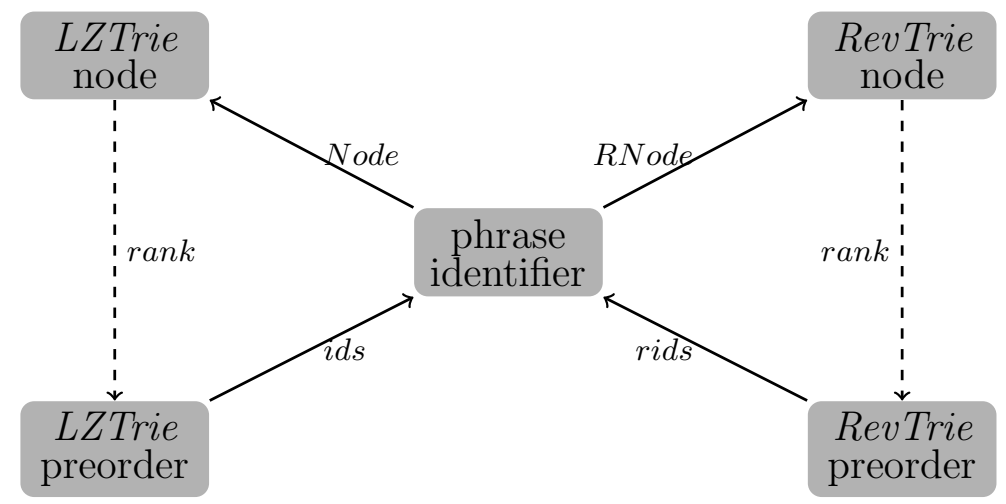

Fig. 6. The original LZ-index navigation structures over index components.

However, we can provide the same navigation functionality needed by the index with a reduced scheme, which we can represent efficiently in the following way:

- LZTrie: the Lempel-Ziv trie, which is implemented with the following data structures

- $\operatorname{par}[0 . .2 n-1]$ : the tree shape of LZTrie represented with DFUDS [6], requiring $2 n+o(n)$ bits.

- letts[1..n]: the array of symbols labeling the edges of LZTrie, represented as explained in Section 2.5 so as to allow operation $\operatorname{child}(x, \alpha)$ in constant time, requiring $n \log \sigma+o(n)$ bits of space. We can get the $i$-th symbol in preorder by first finding the $i$-th node in preorder in par, and then retrieving its symbol as explained in Section 2.5.

- $i d s[1 . . n]$ : the array of LZ78 phrase identifiers in preorder. $i d s[0]=0$ always holds, so we do not store this value. Note that $i d s$ is a permutation of $\{1, \ldots, n\}$, and hence we use the representation of Munro et al. [31] such that the inverse permutation $i d s^{-1}(j)$ can be computed in $O(1 / \epsilon)$ time, requiring $(1+\epsilon) n \log n$ bits, for any $0<\epsilon<1$.

- RevTrie: the Patricia tree [29] of the reversed LZ78 phrases, which is implemented with the following data structures

- $\operatorname{rpar}\left[0 . .2 n^{\prime}-1\right]$ : the RevTrie structure, compressing empty unary paths and represented with DFUDS, thus ensuring $n^{\prime} \leqslant 2 n$ nodes, because empty non-unary nodes still exist. The space requirement is $2 n^{\prime}+o\left(n^{\prime}\right)$ bits to support the same functionalities as LZTrie.

- $\operatorname{rletts}\left[1 . . n^{\prime}\right]$ : the array storing the first symbol of each edge label in RevTrie, represented as for LZTrie and requiring $n^{\prime} \log \sigma+o\left(n^{\prime}\right)$ bits of space. 
- $B\left[1 . . n^{\prime}\right]$ : a bit vector supporting rank and select queries, and requiring $n^{\prime}(1+o(1))$ bits [30]. This bit vector marks the non-empty nodes: the $j$-th bit of $B$ is 1 iff the node with preorder position $j$ in rpar is not empty, otherwise the bit is 0 . Given a position $p$ in rpar corresponding to a RevTrie node, the corresponding bit in $B$ is $B[\operatorname{rank}(\operatorname{rpar}, p-1)]$.

- skips $\left[1 . . n^{\prime}\right]$ : the Patricia tree skips of the nodes in preorder, using $\log \log u$ bits per node and inserting empty unary nodes when the skip exceeds $\log u$. In this way, one out of $\log u$ empty unary nodes could be explicitly represented. In the worst case there are $O(u)$ empty unary nodes, of which $O(u / \log u)$ are explicitly represented. This adds $O(u / \log u)$ nodes to $n^{\prime}$, which translates into $O\left(\left(n^{\prime}+\frac{u}{\log u}\right)(3+\log \sigma+\log \log u)\right)=o(u \log \sigma)$ bits overall for the RevTrie nodes, symbols, and skips.

- R[1..n]: a mapping from RevTrie preorder positions to LZTrie preorder positions. Given a non-empty RevTrie node with preorder $i$ (just counting non-empty nodes), we define the corresponding $L Z$ Trie preorder as $R[i]=i d s^{-1}$ (rids $\left.[i]\right)$. This is a permutation and is represented using the succinct data structure for permutations of Munro et al. [31], requiring $(1+\epsilon) n \log n$ bits to represent $R$ and compute $R^{-1}$ in $O(1 / \epsilon)$ worst-case time. Given a position $p$ in rpar corresponding to a non-empty RevTrie node, the corresponding $R$ value (i.e., preorder in LZTrie) can be computed as $R\left[\operatorname{rank}_{1}\left(B\right.\right.$, rank $_{\text {(rpar, }}$-1) $\left.)\right]$.

In Fig. 7 we draw the navigation scheme. The search algorithm remains the same since we can map preorder positions to nodes in the DFUDS representation of the tries and vice versa (see Section 2.5), and also we can simulate the missing arrays $\operatorname{rids}(i) \equiv i d s[R[i]], R N o d e(i) \equiv$ select) $\left(\operatorname{rpar}, R^{-1}\left(i d s^{-1}(i)\right)\right)+1$, and $\operatorname{Node}(i) \equiv$ select $\left(\right.$ par, $\left.i d s^{-1}(i)\right)+1$, all of which take $O(1 / \epsilon)$ time.

The space requirement is $(2+\epsilon) n \log n+3 n \log \sigma+2 n \log \log u+8 n+o(u)=(2+\epsilon) n \log n+$ $o(u \log \sigma)$ bits, which according to Lemma 1 is $(2+\epsilon) u H_{k}(T)+o(u \log \sigma)$ bits, for any $k=o\left(\log _{\sigma} u\right)$.

The child operation on RevTrie can now be computed in $O(1)$ time thanks to DFUDS, to rletts, and to the skips, versus the $O(h \log \sigma)$ time of the original LZ-index [34]. Now, because RevTrie is a Patricia tree and the underlying strings are not readily available, it is not obvious how to traverse it. The next lemma addresses this issue.

Lemma 3. Given a string $s \in \Sigma^{*}$, we can determine whether it is represented in RevTrie or not (finding the corresponding node in the affirmative case) in $O(|s|)$ time.

Proof. To find the node corresponding to string $s$ we descend from the RevTrie root, using operation $\operatorname{child}(x, \alpha)$ on the first symbol of each edge label, which is stored in rletts, and using the skips to compute the next symbol of $s$ to use in the descent. If $s$ cannot be consumed while descending, then we determine that it is not represented in RevTrie in $O(|s|)$ time. Otherwise, assume that after consuming string $s$ in this way we arrive at node $v_{r}$ with preorder $j$ in RevTrie. The string labeling the root-to- $v_{r}$ path in RevTrie can be computed by accessing the node $v_{l z}$ with preorder $R[j]$ in LZTrie, and then extracting the string labeling the $v_{l z}$-to-root path in LZTrie. Then we compare that string against $s$ to verify that the node we arrived at corresponds to $s$, or otherwise that $s$ does not occur in RevTrie.

In case that node $v_{r}$ in RevTrie is empty, $R[j]$ is undefined. Notice, however, that there must be at least one non-empty node descending from this empty node, since leaves in RevTrie cannot be empty as they always correspond to an LZ78 phrase. Given that the string represented by every non-empty node in the subtree of node $v_{r}$ has the string $s$ as a prefix, the corresponding strings in LZTrie have $s^{r}$ as a suffix. So we can use any $R$ value within the subtree of node $v_{r}$ in order to 
map to the LZTrie and then extract the string it represents. We can compute the preorder $j^{\prime}$ of the next non-empty node within that subtree by $j^{\prime}=\operatorname{select}_{1}\left(B, \operatorname{rank}_{1}(B, j)+1\right)$, where $\operatorname{rank}_{1}(B, j)$ represents the number of non-empty nodes up to node $v_{r}$. Thus, we use $R\left[j^{\prime}\right]$ to access the LZTrie and extract the corresponding string. We know when to stop extracting, since the length of the string represented by $v_{r}$ matches the length of the string we are looking for.

The overall cost for the descending process is therefore $O(|s|)$.

Operations child and parent on LZTrie can be also computed in $O(1)$ time, versus the $O(\log \sigma)$ time of the original LZ-index. Hence, occurrences of type 1 are solved in $O(m+o c c)$ time; occurrences of type 2 are solved now in $O\left(\frac{n}{\epsilon \sigma^{m / 2}}\right)$ average time, where the $O(1 / \epsilon)$ factor comes from simulating Node and RNode (by using $i d s^{-1}$ and $R^{-1}$ respectively) for the checks of type 2 ; occurrences of type 3 are solved in $O\left(\frac{m^{2}}{\epsilon}\right)$ time, since we use the method of Lemma 2 to find the maximal concatenations of phrases in time $O\left(\frac{m^{2}}{\epsilon}\right)$ (because Node is simulated), and then we need to use Node and RNode to check every possible candidate, in $O\left(\frac{m^{2}}{\epsilon}\right)$ time as well. Therefore, the occ occurrences of $P$ can be located in $O\left(\frac{m^{2}}{\epsilon}+\frac{n}{\epsilon \sigma^{m / 2}}\right)$ average time, for $0<\epsilon<1$. This time is $O\left(\frac{m^{2}}{\epsilon}\right)$ on average for $m \geqslant 2 \log _{\sigma} u$.

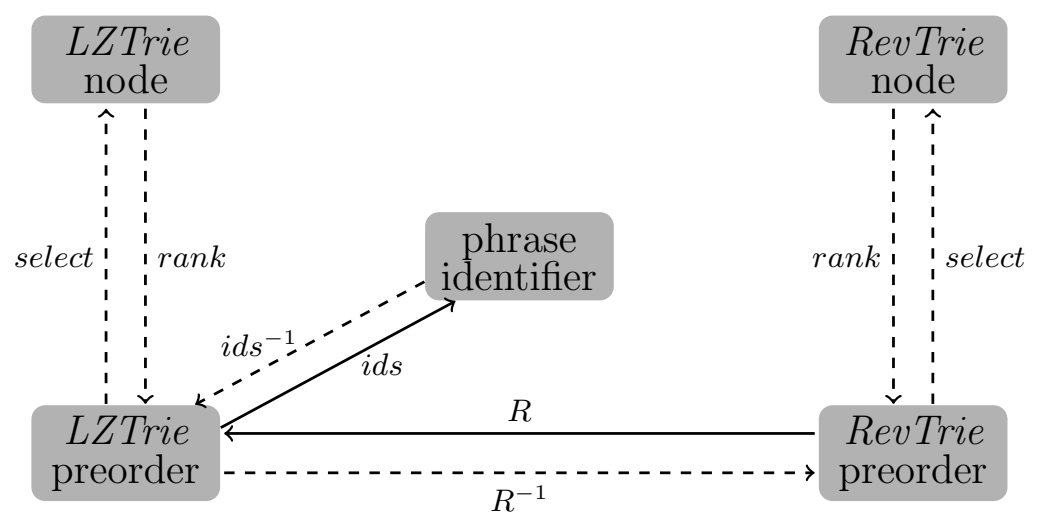

Fig. 7. Reduced navigation scheme over LZ-index components, requiring $(2+\epsilon) u H_{k}+o(u \log \sigma)$ bits.

\section{Suffix Links in RevTrie}

As we have seen in Section 3.4, for the LZ-index we can achieve $(2+\epsilon) u H_{k}(T)+o(u \log \sigma)$ bits of space and $O\left(m^{2}\right)$ average search time for patterns of length $m \geqslant 2 \log _{\sigma} u$. Hence, two questions may arise:

Question 1. Can we reduce the space requirement of LZ-index to $(1+\epsilon) u H_{k}(T)+o(u \log \sigma)$ bits?, i.e., to almost optimal space (in terms of $H_{k}$ ).

Question 2. Can we retain worst-case guarantees at search time (as for the original LZ-index), yet requiring $(2+\epsilon) u H_{k}(T)+o(u \log \sigma)$ bits of storage (as for the scheme of Fig. 7)?

In this section (and in the next one) we will find afirmative answers to these questions. Specifically, Theorem 1 shall answer Question 1, and Theorem 2 shall answer Question 2. 
We will build on the reduced scheme described in Section 3.4 and illustrated in Fig. 7. Given a LZTrie node with preorder position $i$, let us define $\operatorname{parent}_{l z}(i) \equiv \operatorname{rank}$ (par,parent $($ select $)($ par, $i)+$ $1)-1)$. That is, parent $_{l z}$ is the parent operation working on preorders rather than on the corresponding nodes. Let $\operatorname{child}_{l z}(i, \alpha)$ be defined similarly for $\operatorname{child}(x, \alpha)$ operation. Also, let us define $\operatorname{letts}_{l z}(i)=\operatorname{letts}\left[\operatorname{rank}_{(}(\right.$par,parent $\left.(x))-1+\operatorname{childrank}(x)-1\right]$, where node $x$ is computed as select, $($ par, $i)+1$, which yields the symbol by which the node with preorder $i$ descends from its parent. We denote $\operatorname{str}_{l z}(i)$ the string represented by node with preorder $i$ in LZTrie. In the same way we define $\operatorname{str}_{r}(j)$ for node with preorder $j$ in RevTrie.

The idea is that we are going to compress the $R$ mapping defined for that version of the LZ-index. Let us see this array as a kind of suffix array which, instead of storing text positions, stores LZTrie preorder positions (see Section 2.4). Array $R$ is a lexicographically sorted array of the reversed LZ78 phases (because it is sorted according to RevTrie preorders). Given a reversed phrase with preorder $i$ in RevTrie (and preorder $R[i]$ in LZTrie), its longest proper suffix has position parent $t_{l z}(R[i])$ in LZTrie (as this corresponds to the longest proper prefix in LZTrie). Given a reverse phrase with position $j$ in LZTrie, its lexicographic rank is $R^{-1}[j]$.

Given this analogy, the question is: can we compress the $R$ mapping just as we can compress a suffix array $[18,39]$ ? Therefore, we define now the analogue in LZ-index to function $\Psi$ of Compressed Suffix Arrays (recall Section 2.4).

Definition 4. For every RevTrie preorder $1 \leqslant i \leqslant n$ we define function $\varphi$ such that $\varphi(i)=$ $R^{-1}\left(\right.$ parent $\left._{l z}(R[i])\right)$, and $\varphi(0)=0$.

We have the following properties for function $\varphi$.

Property 4. Given a non-empty node with preorder $i$ in RevTrie, such that $\operatorname{str}_{r}(i)=a x$, for some $a \in \Sigma, x \in \Sigma^{*}$, then

1. $\operatorname{str}_{r}(\varphi(i))=x$,

2. $R[\varphi(i)]=$ parent $_{l z}(R[i])$, and

3. letts $_{l z}(R[i])=a$.

Point 1 means that $\varphi$ acts as a suffix link in RevTrie, and it follows from the fact that since $\operatorname{str}_{r}(i)=$ $a x$, then $\operatorname{str}_{l z}(R[i])=x^{r} a$, because node $i$ in RevTrie corresponds to node $R[i]$ in LZTrie. Therefore, $\operatorname{str}_{l z}\left(\operatorname{parent}_{l z}(R[i])\right)=x^{r}$, which finally means $\operatorname{str}_{r}\left(R^{-1}\left(\operatorname{parent}_{l z}(R[i])\right)\right)=\operatorname{str}_{r}(\varphi(i))=x$. Point 2 implies that by following a suffix link in RevTrie, we are "going to the parent" in LZTrie, and it follows from applying $R$ to both sides of the equation in Definition 4 . Fig. 8 illustrates. Note that the edge connecting the LZTrie nodes with preorders $R[\varphi(i)]$ and $R[i]$ is labelled $a$ (as stated by point 3 in Property 4) which is the same symbol we are missing when following the suffix link $\varphi(i)$ in RevTrie.

We can prove that RevTrie is suffix closed since LZTrie is prefix closed, hence suffix links are well defined.

Lemma 4. Every non-empty node in RevTrie has a suffix link.

Proof. Let us consider any non-empty node in RevTrie with preorder $i$, such that $\operatorname{str}_{r}(i)=a x$, for $a \in \Sigma$ and $x \in \Sigma^{*}$. As ax is a RevTrie phrase (with preorder $i$ ), then $x^{r} a$ must be a LZTrie phrase (with preorder $R[i]$ ). By Property 1 of the LZ78 parsing it follows that $x^{r}$ is also a LZTrie phrase and thus $x$ must be a RevTrie phrase. Hence, every non-empty node in RevTrie (i.e., every RevTrie node belonging to a reverse LZ78 phrase) has a suffix link. 
We will use Property 4 to reduce the space requirement of the $R$ mapping: suppose that we do not store $R[i]$, for the RevTrie node with preorder $i$ in Fig. 8, but we store $R[\varphi(i)]$; then note that $R[i]$ can be computed as $\operatorname{child}_{l z}(R[\varphi(i)], a)$. Russo and Oliveira [38] also use properties of suffix links in their index; however, their main objective is to reduce the locating complexity of their LZ-index to $O((m+o c c) \log u)$, yet not being able of reducing the space requirement as we do. In Section 6, however, we will show how to use suffix links to reduce the time complexity of our indices, achieving their same locating complexity while requiring less space.

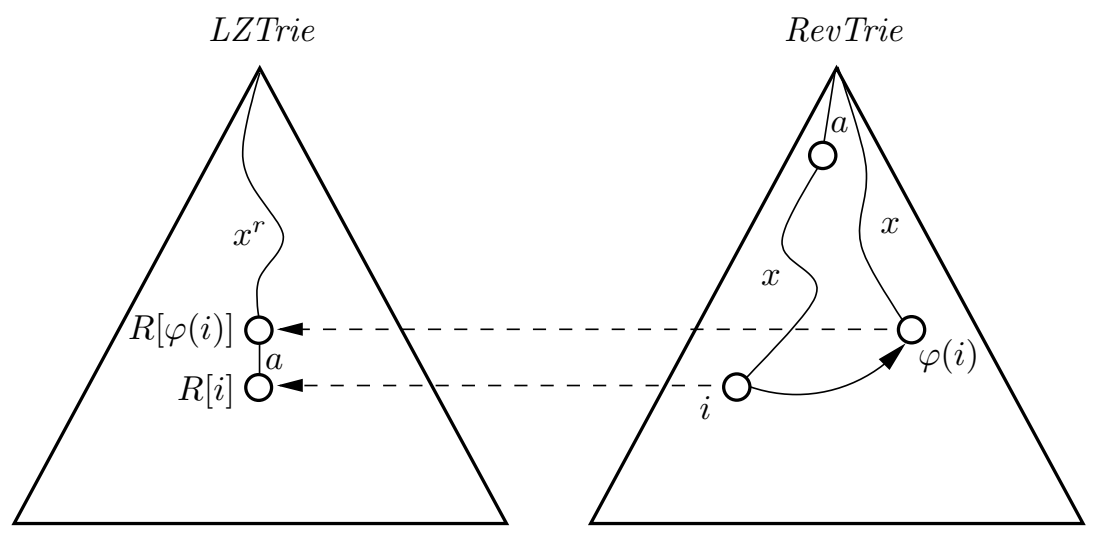

Fig. 8. Illustration of Property 4. Preorder numbers, both in LZTrie and RevTrie, are shown outside each node. Dashed arrows associate a RevTrie node with its corresponding node in LZTrie. This association is given by the $R$ mapping.

In Table 3 we show some arrays conforming the reduced version of LZ-index for our running example, including the $\varphi$ function and the set of reversed LZ78 phrases in RevTrie (in preorder, i.e., lexicographically sorted).

\subsection{Using Suffix Links to Compute $R$}

Let us show how to compute $R[i]$ using function $\varphi$. We define array $L[1 . . n]$, which for each nonempty node with preorder $i$ in RevTrie stores the first symbol of the string $\operatorname{str}_{r}(i)$. In the RevTrie of Fig. 5(b), it holds that $L[i]=$ 'a' for every $i$ in the preorder interval $[2,5]$. In the same example, note that if we follow the suffix link $\varphi(i)$, for $2 \leqslant i \leqslant 5$, we discard the symbol ' $a$ '.

In the example of Fig. 8 we have $L[i]=a$; as we said before, this also means that $L[i]$ is the label of the edge connecting LZTrie nodes with preorders $R[\varphi(i)]$ and $R[i]$. In other words, $L[i]=\operatorname{letts}_{l z}(R[i])$. In Table 3 we show the values of $L$ for our example.

It is not hard to prove that $L[i] \leqslant L[j]$ whenever $i \leqslant j$ : let $i$ and $j$ be two preorders in RevTrie, such that $i \leqslant j$. Therefore, for the strings corresponding to these preorders it holds that $\operatorname{str}_{r}(i) \leqslant \operatorname{str}_{r}(j)$. As $L[i]$ and $L[j]$ store the first symbol of $\operatorname{str}_{r}(i)$ and $\operatorname{str}_{r}(j)$ respectively, then it holds that $L[i] \leqslant L[j]$. Thus, $L$ can be divided into $\sigma$ runs of equal symbols. In this way $L$ can be represented by an array $L^{\prime}$ of at most $\sigma \log \sigma$ bits and a bit vector $L_{B}$ of $n+o(n)$ bits, such that $L_{B}[i]=1$ iff $L[i] \neq L[i-1]$, for $i=2 \ldots n$, and $L_{B}[1]=0$ (this position belongs to the text terminator "\$", which is not in the alphabet). For every $i$ such that $L_{B}[i]=1$, we store $L^{\prime}\left[\operatorname{rank}_{1}\left(L_{B}, i\right)\right]=L[i]$. Hence, $L[i]$ can be computed as $L^{\prime}\left[\operatorname{rank}_{1}\left(L_{B}, i\right)\right]$ in $O(1)$ time. 
Table 3. Illustration of the different components of our index for the running example. In the case of RevTrie, array rids is shown just for simplicity, yet this is not explicitly stored. In each case, $i$ indicates the preorders in each trie.

\begin{tabular}{cccc}
\multicolumn{4}{c}{ LZTrie components } \\
\hline$i$ & $i d s[i]$ & letts $_{l z}(i)$ & $R^{-1}(i)$ \\
\hline 0 & 0 & & 0 \\
1 & 1 & $\mathrm{a}$ & 2 \\
2 & 17 & $\$$ & 1 \\
3 & 3 & $\mathrm{~b}$ & 6 \\
4 & 15 & $\mathrm{r}$ & 15 \\
5 & 14 & $\mathrm{l}$ & 10 \\
6 & 4 & $\mathrm{r}$ & 14 \\
7 & 12 & $\mathrm{a}$ & 4 \\
8 & 10 & $\mathrm{~d}$ & 8 \\
9 & 16 & $\mathrm{l}$ & 11 \\
10 & 6 & - & 17 \\
11 & 11 & $\mathrm{p}$ & 13 \\
12 & 2 & $\mathrm{l}$ & 9 \\
13 & 7 & $\mathrm{a}$ & 3 \\
14 & 9 & $\mathrm{~b}$ & 7 \\
15 & 5 & - & 16 \\
16 & 8 & $\mathrm{a}$ & 5 \\
17 & 13 & $\mathrm{p}$ & 12 \\
\hline
\end{tabular}

\begin{tabular}{ccccccl}
\hline \multicolumn{7}{c}{ RevTrie components } \\
\hline 0 & rids $[i]$ & $R[i]$ & $\varphi(i)$ & $L[i]$ & $L_{B}[i]$ & string in RevTrie \\
\hline 1 & 17 & 2 & 2 & $\$$ & 0 & \$a \\
\hline 2 & 1 & 1 & 0 & a & 1 & a \\
3 & 7 & 13 & 9 & a & 0 & al \\
4 & 12 & 7 & 14 & a & 0 & ara \\
5 & 8 & 16 & 16 & a & 0 & a_- \\
\hline 6 & 3 & 3 & 2 & b & 1 & ba \\
7 & 9 & 14 & 3 & b & 0 & bal \\
\hline 8 & 10 & 8 & 14 & d & 1 & dra \\
\hline 9 & 2 & 12 & 0 & l & 1 & l \\
10 & 14 & 5 & 2 & 1 & 0 & la \\
11 & 16 & 9 & 14 & 1 & 0 & lra \\
\hline 12 & 13 & 17 & 5 & p & 1 & pa_ \\
13 & 11 & 11 & 17 & p & 0 & p_a \\
\hline 14 & 4 & 6 & 2 & r & 1 & ra \\
15 & 15 & 4 & 6 & r & 0 & rba \\
\hline 16 & 5 & 15 & 0 & - & 1 & - \\
17 & 6 & 10 & 2 & - & 0 & -a \\
\hline
\end{tabular}

Given a RevTrie preorder position $i$, in order to compute $R[i]$ we could follow suffix links in RevTrie starting from node with preorder $i$, until we reach the RevTrie root. At this point we could apply, starting from the root of LZTrie, child operations using the first symbol of each RevTrie string we got while following suffix links, in reverse order. This procedure is formalized in the following lemma.

Lemma 5. Given a RevTrie preorder position $0 \leqslant i \leqslant n$, the corresponding LZTrie preorder position $R[i]$ can be computed by the following recurrence:

$$
R[i]= \begin{cases}\operatorname{child}_{l z}(R[\varphi(i)], L[i]) & \text { if } i \neq 0 \\ 0 & \text { if } i=0\end{cases}
$$

Proof. $R[0]=0$ holds from the fact that the preorder position corresponding to the empty string, both in LZTrie and RevTrie, is 0. To prove the other part we note that if $x$ is the parent in LZTrie of node $y$ with preorder position $R[i]$, then the symbol labeling the edge connecting $x$ to $y$ is stored in $L[i]=\operatorname{letts}_{l z}(R[i])$. That is, $\operatorname{child}_{l z}\left(\operatorname{parent}_{l z}(R[i]), L[i]\right)=R[i]$. The lemma follows from this fact and replacing $\varphi(i)$ by Definition 4 in the recurrence.

Example 2. To compute $R[13]$ for the example of Table 3, which corresponds to string 'p_a' in RevTrie, we need to compute $\operatorname{child}_{l z}(R[17], L[13])$, where $L[13]=$ 'p' and $\varphi(13)=17$ corresponds to the RevTrie preorder position of string '_a'. Now, to compute $R[17]$ we need to compute $\operatorname{child}_{l z}(R[2], L[17])$, where $L[17]=$ '.'. Then, $R[2]$ is computed as $\operatorname{child}_{l z}(R[0], L[2])$, which is just child(0, 'a'). At this point we must perform the child operations from the LZTrie root. Recall that we assume the child $_{l z}$ operation works on preorder positions, so we must compute $\operatorname{child}_{l z}\left(\operatorname{child}_{l z}\left(\operatorname{child}_{l z}\left(0\right.\right.\right.$, 'a'$\left.\left.^{\prime}\right),{ }^{\prime}-'\right)$, 'p') in LZTrie, which is the same as child $d_{l z}\left(\operatorname{child}_{l z}\left(1,{ }^{\prime}-'\right)\right.$, 'p'), which in turn is child $_{l z}\left(10,{ }^{\prime} \mathrm{p}\right.$ '), which finally yields the node with preorder position 11 . Hence, we conclude that $R[13]=11$. 


\subsection{Compressing the $R$ Mapping}

As in the case of the $\Psi$ function of Compressed Suffix Arrays [18,39], we can prove the following lemma for the $\varphi$ function, which is the key to compress the $R$ mapping.

Lemma 6. For every $i<j$, if $L[i]=L[j]$, then $\varphi(i)<\varphi(j)$.

Proof. Let $\operatorname{str}_{r}(i)$ denote the $i$-th string in the lexicographically sorted set of reversed strings. Note that $\operatorname{str}_{r}(i)<\operatorname{str}_{r}(j)$ iff $i<j$. If $i<j$ and $L[i]=L[j]$ (i.e., $\operatorname{str}_{r}(i)$ and $\operatorname{str}_{r}(j)$ start with the same symbol), then $\operatorname{str}_{r}(\varphi(i))<\operatorname{str}_{r}(\varphi(j))$ (as $\operatorname{str}_{r}(\varphi(i))$ is $\operatorname{str}_{r}(i)$ without its first symbol, recall Property 4, point 1), and thus $\varphi(i)<\varphi(j)$.

Corollary 1. Array $\varphi$ can be partitioned into at most $\sigma$ strictly increasing sequences.

This fact is illustrated in Table 3, where the increasing runs of $\varphi$, corresponding to runs of equal symbols in $L$, are separated by horizontal lines.

As a result, we replace $R$ by $\varphi, L^{\prime}$, and $L_{B}$, and use them to compute a given value $R[i]$. According to Corollary 1, we can represent $\varphi$ using the idea of Sadakane [39] to represent $\Psi$, which was explained in Section 2.4. Thus, $\varphi$ can be encoded with $n H_{0}($ letts $)+O(n \log \log \sigma)$ bits, and hence we replace the $n \log n$-bits representation of $R$ by the $n H_{0}($ letts $)+O(n \log \log \sigma)+n+o(n)=$ $O(n \log \sigma)=o(u \log \sigma)$ bits of the representation of $\varphi, L^{\prime}$, and $L_{B}$. The absolute values of $\varphi$ now add $O(n)$ bits, while the size of the table used to sum the differences is $o(n)$ bits.

\subsection{Computing $R$ in $O(1 / \epsilon)$ Time}

The time to compute $R[i]$ according to Lemma 5 is $O\left(\left|s t r_{r}(i)\right|\right)$, which actually corresponds to traversing LZTrie from the root with the symbols of $s t_{r}(i)$ in reverse order. However, the procedure of Lemma 5 can be adapted to allow constant-time computation of $R[i]$. We store $\epsilon n$ values of $R$ in an array $R^{\prime}$, plus a bit vector $R_{B}$ of $n+o(n)$ bits indicating which values of $R$ have been stored, ensuring that $R[i]$ can be computed in $O(1 / \epsilon)$ time and requiring $\epsilon n \log n$ extra bits.

To determine the $R$ values to be explicitly stored, we fix $l=\Theta(1 / \epsilon)$ and carry out a post-order traversal on LZTrie and mark the nodes whose height is $j \cdot l$, for some $j>0$. In this way, for every marked node $x$ there is a path of length $l$ with no marked nodes descending from $x$. This path is part of the longer path leading to the farthest leaf $y$ descending from $x$, which determines the height of $x$. Since the height of the nodes lying in the path of length $l$ descending from $x$ is determined by the same leaf $y$, there cannot be any marked node in this path. Note that by using this procedure we can have, for example, paths in the trie which are entirely formed by marked nodes. However, since for every such marked node we have at least $l$ non-marked nodes, we mark $O(\epsilon n)$ nodes overall. We also ensure that, if we start at an arbitrary node in LZTrie and go successively to the parent, in the worst case we must apply $O(1 / \epsilon)$ parent operations to find a marked node. On the RevTrie side, this means that in the worst case we must follow $O(1 / \epsilon)$ suffix links to find a node whose $R$ value has been stored.

If the node to mark is at preorder position $j$, then we set $R_{B}\left[R^{-1}(j)\right]=1$ (note that $R_{B}$ is indexed by RevTrie preorder). After we mark the positions of $R$ to be stored, we scan $R_{B}$ sequentially from left to right, and for every $i$ such that $R_{B}[i]=1$, we set $R^{\prime}\left[\operatorname{rank}_{1}\left(R_{B}, i\right)\right]=R[i]$. Then, we free $R$ since $R[i]$ can be computed in $O(1 / \epsilon)$ worst-case time, as stated by the following lemma. 
Lemma 7. Given a RevTrie preorder position $0 \leqslant i \leqslant n$ and given any constant $0<\epsilon<1$, the corresponding LZTrie preorder position $R[i]$ can be computed in constant $O(1 / \epsilon)$ worst-case time by the following recurrence:

$$
R[i]= \begin{cases}\operatorname{child}\left(R[\varphi(i)], L^{\prime}\left[\operatorname{rank}_{1}\left(L_{B}, i\right)\right]\right) & \text { if } R_{B}[i]=0 \\ R^{\prime}\left[\operatorname{rank}_{1}\left(R_{B}, i\right)\right] & \text { if } R_{B}[i]=1\end{cases}
$$

requiring $\epsilon n \log n+O(n \log \sigma)=\epsilon H_{k}(T)+o(u \log \sigma)$ bits of space.

Note that the same structure used to compute $R^{-1}$ using the explicit representation of $R$ can be used under this reduced-space representation of $R$, with cost $O\left(1 / \epsilon^{2}\right)$ (as we have to access $O(1 / \epsilon)$ positions in $R$ ).

As now we store $i d s$ in $n \log n$ bits, $i d s^{-1}, R^{-1}$, and $R^{\prime}$ in $\epsilon n \log n$ bits, and $\varphi$, letts, and rletts in $O(n \log \sigma)=o(u \log \sigma)$ bits, the total space requirement is $(1+\epsilon) n \log n+o(u \log \sigma)$ bits (renaming $3 \epsilon=\epsilon$ ), and we provide the same navigation scheme as in Fig. 7. Occurrences of type 1 are found as usual, in $O\left(m+\frac{o c c_{1}}{\epsilon}\right)$ time, where the extra $O\left(\frac{o c c_{1}}{\epsilon}\right)$ term appears because we have to use $R$ to map from RevTrie to LZTrie, which takes $O(1 / \epsilon)$ each time. Occurrences of type 2 are solved as explained in Section 3.4, in $O\left(\frac{n}{\epsilon^{2} \sigma^{m / 2}}\right)$ average time since now the access between tries is provided by $R$ and $R^{-1}$, the latter with cost $O\left(1 / \epsilon^{2}\right)$. Finally, for solving occurrences of type 3, we first search for all the pattern substrings in LZTrie in $O\left(\mathrm{~m}^{2}\right)$ time, then compute the maximal concatenations of phrases, in $O\left(\frac{m^{2}}{\epsilon}\right)$ time by using the improved algorithm of Lemma 2 (the $O(1 / \epsilon)$ factor comes from the fact that we use $i d s^{-1}$ to simulate Node), and finally for each of the $O\left(m^{2}\right)$ maximal concatenation found we carry out the tests as explained in Section 3.4, with $\operatorname{cost} O\left(\frac{m^{2}}{\epsilon^{2}}\right)$ because $R$ Node is implemented by using $R^{-1}$. We have proved:

Theorem 1. There exists a compressed full-text self-index requiring $(1+\epsilon) u H_{k}(T)+o(u \log \sigma)$ bits of space, for $\sigma=O(\operatorname{polylog}(u))$, any $k=o\left(\log _{\sigma} u\right)$ and any $0<\epsilon<1$, able to locate (and count) the occ occurrences of a pattern $P[1 . . m]$ in a text $T[1 . . u]$ in $O\left(\frac{m^{2}}{\epsilon^{2}}+\frac{u}{\epsilon^{2} \sigma^{m / 2}}\right)$ average time, which is $O\left(\frac{m^{2}}{\epsilon^{2}}\right)$ if $m \geqslant 2 \log _{\sigma} u$.

Now we can get worst-case guarantees in the search process by adding Range, the two-dimensional range search data structure defined in Section 3 for the original LZ-index, requiring $n \log n+$ $o(u \log \sigma)$ extra bits [25]. Occurrences of type 2 can now be solved in $O\left(\left(m+o c c_{2}\right) \log n\right)$ worst-case time by using Range. Occurrences of type 1 and type 3 are found as for the index of Theorem 1 . Existential queries, on the other hand, can be solved by first looking whether there is any occurrence of type 1 (i.e., by looking for $P^{r}$ in RevTrie and then checking whether the corresponding subtree is empty or not) in $O(m)$ time. If there are no occurrences of type 1 , we check whether there is any occurrence of type 2 by partitioning the pattern and using Range to count the number of occurrences for each partition. This takes $O(m \log n)$ time overall, since we use Range just to count. Finally, if there are no occurrences of type 2, we look for occurrences of type 3 in $O\left(\mathrm{~m}^{2}\right)$ time. Hence, we have the following theorem.

Theorem 2. There exists a compressed full-text self-index requiring $(2+\epsilon) u H_{k}(T)+o(u \log \sigma)$ bits of space, for $\sigma=O(\operatorname{polylog}(u))$, any $k=o\left(\log _{\sigma} u\right)$ and any $0<\epsilon<1$, which is able to: locate the occ occurrences of a pattern $P[1 . . m]$ in a text $T[1 . . u]$ in $O\left(\frac{m^{2}}{\epsilon^{2}}+(m+o c c) \log u+\frac{o c c}{\epsilon}\right)=O\left(m^{2}+(m+\right.$ occ) $\log u$ ) worst-case time; count the number of pattern occurrences in time $O\left(m^{2}+m \log u+o c c\right)$; and determine whether pattern $P$ exists in $T$ in $O\left(m^{2}+m \log u\right)$ time. 
In Section 8 we show that the theorem is valid for the more general case $\log \sigma=o(\log u)$. We leave for Section 7 the study of display and extract queries on our indices.

\section{Using the $x b w$ Transform to Represent the LZTrie}

A different idea to reduce the space requirement of LZ-index is to use the $x b w$ transform of Ferragina et al. [11] to represent the LZTrie. We show that subpath queries, which are efficiently solved by the $x b w$ transform (see Section 2.5), are so powerful that we can carry out the work of both LZTrie and RevTrie only with the $x b w$ representation of LZTrie, thus achieving the same result as in Section 4 (always assuming $\sigma=O($ polylog $(u))$ ), yet by very different means. Ferragina et al. [11] have shown how the $x b w$ representation can be compressed in order to take advantage of the tree regularities, which can be very important in practice and adds extra value to this representation.

\subsection{Index Definition}

We represent LZ-index with the following data structures:

- xbw LZTrie: the $x b w$ representation [11] of the LZTrie, where the nodes are lexicographically sorted according to their upward paths in the trie. We store

- $S_{\alpha}$ : the array of symbols labeling the edges of the trie. In the worst case LZTrie has $2 n$ nodes (because of the dummy leaves we add, recall Section 2.6). We represent this array by using a data structure for rank and select [13], which are needed to compute the operations on $x b w$. The space requirement is $2 n \log \sigma+o(n \log \sigma)$ bits.

- $S_{\text {last }}$ : a bit array such that $S_{\text {last }}[i]=1$ iff the corresponding node in LZTrie is the last child of its parent. We represent this array with a data structure for rank and select [30]. The space requirement is at most $2 n+o(n)$ bits.

See Table 2 for an illustration of the $x b w$ of LZTrie for the running example.

- Balanced parentheses LZTrie: the trie of the Lempel-Ziv phrases, implemented by

- par: the balanced parentheses representation [32] of LZTrie. In order to index the LZTrie leaves with $x b w$, we have to add a dummy child to each, as it was explained in Section 2.5. In this way, the trie has $n^{\prime} \leqslant 2 n$ nodes. Non-dummy nodes are marked in a bit vector $B\left[1 . . n^{\prime}\right]$ in the same way as empty nodes are marked in RevTrie (see Section 3.4). We represent array $B$ with a data structure for rank and select queries [30]. The space requirement is $2 n^{\prime}+n^{\prime}+o(n)$ bits, which is $6 n+o(n)$ bits in the worst case. This sequence par is needed to solve some operations which are not supported by the $x b w$, such as ancestor $(x, y)$ and $\operatorname{depth}(x)$.

- ids: the array of LZ78 phrase identifiers in preorder, only for non-dummy nodes (we find the phrase identifier for a given node by using $\operatorname{rank}_{1}$ on $B$ ). This array is represented by the data structure of Munro et al. [31], such that we can compute the inverse permutation $i d s^{-1}$ in $O(1 / \epsilon)$ time, requiring $(1+\epsilon) n \log n$ bits.

See Fig. 9 for an illustration.

- Pos: a mapping from $x b w$ positions to the corresponding LZTrie preorder positions (i.e., this is a permutation of LZTrie preorders). See Fig. 9 for an illustration. In the worst case there are $2 n$ such positions, and so the space requirement is $2 n \log (2 n)$ bits. We can reduce this space to $\epsilon n \log (2 n)$ bits by storing in an array $\mathrm{Pos}^{\prime}$ one out of $O(1 / \epsilon)$ values of Pos, such that $P o s[i]$ can be computed in $O(1 / \epsilon)$ time. We need a bit vector $\operatorname{Pos}_{B}$ of $2 n+o(n)$ bits indicating 
which values of Pos have been stored. Assume we need to compute the preorder position Pos $[i]$, for a given $x b w$ position $i$. If $\operatorname{Pos}_{B}[i]=1$, then such preorder position is stored explicitly at $\operatorname{Pos}^{\prime}\left[\operatorname{rank}_{1}\left(\operatorname{Pos}_{B}, i\right)\right]$. Otherwise, we simulate a preorder traversal in $x b w$ from the node at $x b w$ position $i$, until $\operatorname{Pos}_{B}[j]=1$, for an $x b w$ position $j$. Each preorder step we perform in $x b w$ corresponds to moving to the next opening parenthesis in par. Once this $j$ is found, we map to the preorder position $j^{\prime}=\operatorname{Pos}^{\prime}\left[\operatorname{rank}_{1}\left(\operatorname{Pos}_{B}, j\right)\right]$. If $d$ is the number of nodes in preorder traversal from $x b w$ position $i$ to $x b w$ position $j$, then $j^{\prime}-d$ is the preorder position corresponding to the node at $x b w$ position $i$. We also need to compute $P_{o s}{ }^{-1}$, which can be done in $O\left(1 / \epsilon^{2}\right)$ time under this scheme, requiring $\epsilon n \log (2 n)$ extra bits if we use the representation of [31] for inverse permutations.

- Range: a range search data structure in which we store the point $k$ (belonging to phrase identifier $k$ ) at coordinate $(x, y)$, where $x$ is the $x b w$ position of node for phrase $k$ and $y$ is the LZTrie preorder position of node for phrase $k+1$. We use the data structure of Chazelle [8], as for the original LZ-index. The space requirement is $n \log n+O(n \log \log n)=n \log n+o(u \log \sigma)$ bits.

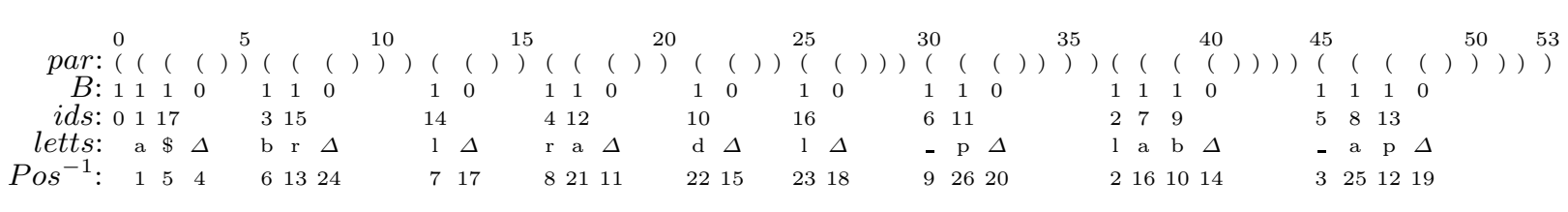

\begin{tabular}{rllllllllllllllllllllllllll}
\hline$i:$ & 1 & 2 & 3 & 4 & 5 & 6 & 7 & 8 & 9 & 10 & 11 & 12 & 13 & 14 & 15 & 16 & 17 & 18 & 19 & 20 & 21 & 22 & 23 & 24 & 25 & 26 \\
\hline $\operatorname{Pos}[i]:$ & 1 & 19 & 23 & 3 & 2 & 4 & 7 & 16 & 16 & 11 & 25 & 5 & 22 & 13 & 20 & 8 & 15 & 26 & 18 & 10 & 12 & 14 & 6 & 24 & 17 \\
\hline
\end{tabular}

Fig. 9. Balanced-parentheses representation of LZTrie for the running example, with dummy leaves added in order to index the (original) leaves with the $x b w$ representation. Bit vector $B$ marks with a 0 the dummy nodes. Given node $x$, the corresponding phrase identifier can be computed as $i d s\left[\operatorname{rank}_{1}(B, \operatorname{preorder}(x))\right]$. We also show the Pos mapping (from $x b w$ positions, see Table 2, to LZTrie preorders), and the Pos $^{-1}$ mapping (from LZTrie preorders to $x b w$ positions).

In Fig. 10 we show the basic resulting navigation scheme following the notation of Section 3.4. The total space requirement is $(2+\epsilon) n \log n+2 n \log \sigma+10 n+O(n \log \log n)+o(n)$ bits, which is $(2+\epsilon) u H_{k}(T)+o(u \log \sigma)$ bits for $k=o\left(\log _{\sigma} u\right)$.

\subsection{Search Algorithm}

We depict now the search algorithm for a pattern $P$ of length $m$.

Occurrences of Type 1. Recall from Section 3.3 that first we need to find all phrases having $P$ as a suffix. To do this we perform a subpath query with $P^{r}$ on the $x b w$ representation of LZTrie, simulating in this way the work done on RevTrie in the original scheme, in $O(m)$ time. Suppose that we obtain the interval $\left[x_{1} . . x_{2}\right]$ in the $x b w$ of LZTrie, corresponding to all nodes whose phrase ends with $P$. In other words, the interval $\left[x_{1} \ldots x_{2}\right]$ contains the roots of the subtrees containing the nodes we are looking for to solve occurrences of type 1 . For each position $i \in\left[x_{1} \ldots x_{2}\right]$, we can get the corresponding preorder in the parentheses representation using $\operatorname{Pos}(i)$, which takes $O(1 / \epsilon)$ time, and then select $($ par, Pos $(i))$ yields the node position. As in the worst case this mapping is carried 


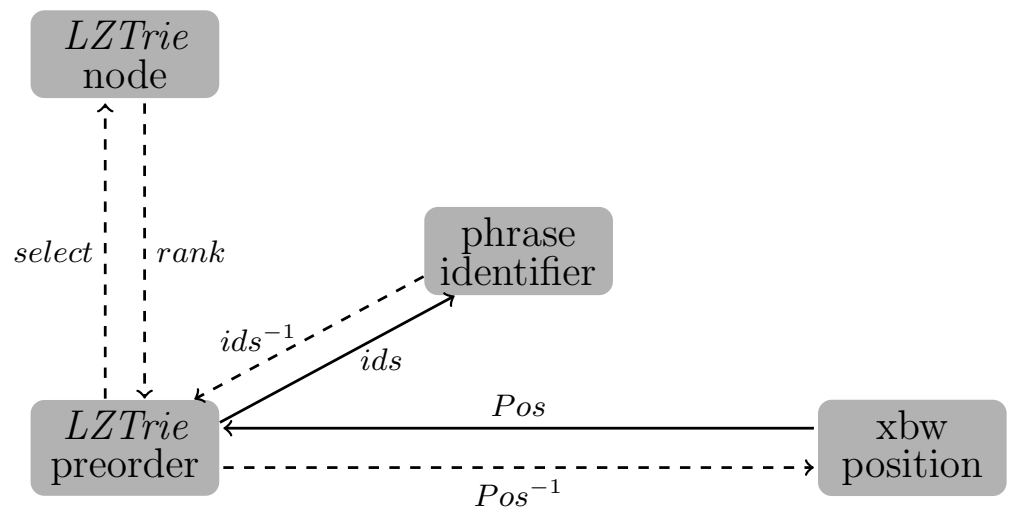

Fig. 10. Basic navigation scheme for the scheme using the $x b w$ representation of LZTrie.

out occ times, the overall time is $O\left(\frac{o c c}{\epsilon}\right)$. Finally we traverse the subtrees of these nodes in par and report all the identifiers found, in constant time per occurrence as done with the usual LZ-index.

Occurrences of Type 2. To solve occurrences of type 2, for every possible partition $P[1 . . i]$ and $P[i+1 . . m]$ of $P$, we traverse the $x b w$ from the root, using operation $\operatorname{child}(x, \alpha)$ with the symbols of $P[i+1 . . m]$. This takes $O\left(m^{2}\right)$ time overall for the $m-1$ partitions of $P$. In this way we are simulating the work done on LZTrie when solving occurrences of type 2 in the original scheme. Once this is found, say at $x b w$ position $j$, we switch to the preorder tree (parentheses) using $\operatorname{select}_{(}(\operatorname{par}, \operatorname{Pos}(j))$, to get the node $v_{l z}$ whose subtree has preorder interval $\left[y_{1} . . y_{2}\right]$ of all the nodes that start with $P[i+1 . . m]$. This takes overall $O\left(\frac{m}{\epsilon}\right)$ time, for the $m-1$ partitions of $P$. Next we perform a subpath query for $P[1 . . i]$ in $x b w$, and get the $x b w$ interval $\left[x_{1} \ldots x_{2}\right]$ of all the nodes that finish with $P[1 . . i]$ (actually we have to perform $x_{1} \leftarrow \operatorname{rank}_{1}\left(S_{\text {last }}, x_{1}\right)$ and $x_{2} \leftarrow \operatorname{rank}_{1}\left(S_{\text {last }}, x_{2}\right)$ to avoid counting the same node multiple times, see [11]). This also takes $O\left(m^{2}\right)$ overall. Finally, we search the Range data structure for $\left[x_{1} . . x_{2}\right] \times\left[y_{1} . . y_{2}\right]$ to get all phrase identifiers $t$ such that phrase $B_{t}$ finishes with $P[1 . . i]$ and phrase $B_{t+1}$ starts with $P[i+1 . . m]$, in $O((m+o c c) \log n)$ time overall.

Occurrences of Type 3. For occurrences of type 3, one proceeds mostly as with the original LZTrie (navigating the $x b w$ instead), so as to find all the nodes equal to substrings of $P$ in $O\left(m^{2}\right)$ time. Then, for each maximal concatenation of phrases $P[i . . j]=B_{t} \ldots B_{\ell}$ we must check that phrase $B_{\ell+1}$ starts with $P[j+1 . . m]$ and that phrase $B_{t-1}$ finishes with $P[1 . . i-1]$. The first check can be done in $O(1 / \epsilon)$ time by using $i d s^{-1}$ : as we have searched for all substrings of $P$ in the trie, we know the preorder interval of the descendants of $P[j+1 . . m]$, thus we check whether the node at preorder position $i d s^{-1}(\ell+1)$ belongs to that interval. The second check can be done in $O\left(1 / \epsilon^{2}\right)$ time, by determining whether $t-1$ lies in the $x b w$ interval of $P[1 . . i-1]$ (that is, $B_{t-1}$ finishes with $P[1 . . i-1])$. For this, we need $\operatorname{Pos}^{-1}$, so that the position is $\operatorname{Pos}^{-1}\left(i d s^{-1}(t-1)\right)$.

Summarizing, occurrences of type 1 cost $O\left(m+\frac{o c c}{\epsilon}\right)$, occurrences of type 2 cost $O\left(m^{2}+\frac{m}{\epsilon}+\right.$ $(m+o c c) \log n)$, and type 3 cost $O\left(\frac{m^{2}}{\epsilon^{2}}\right)$. Thus, we have achieved Theorem 2 again with radically different means. The same complexities are also achieved for counting and existencial queries. We can also get a version requiring $(1+\epsilon) u H_{k}(T)+o(u \log \sigma)$ bits and $O\left(m^{2}\right)$ average reporting time 
if $m \geqslant 2 \log _{\sigma} n$ (as in Theorem 1 ) if we solve occurrences of type 2 by using a procedure similar to that used to solve occurrences of type 3 .

\section{$6 \quad$ Faster and Still Small LZ-indices}

In Section 4 we have shown how to use suffix links in RevTrie to reduce the space requirement of LZ-index. Russo and Oliveira [38] show how to use suffix links to reduce the locating time of their LZ-index to $O((m+o c c) \log u)$; yet, they do not use suffix links to reduce the space of their index. On the other hand, Ferragina and Manzini [12] combine the backward-search concept with a Lempel-Ziv-based scheme to achieve optimal $O(m+o c c)$ locating time, without restrictions on $m$ or occ. Yet, their index is even larger, requiring $O\left(u H_{k}(T) \log ^{\gamma} u\right)$ bits of space, for any constant $\gamma>0$.

In this section we use suffix links to speed up occurrences of type 2, using an idea similar to that of [38], and we solve occurrences of type 3 as a particular case of occurrences of type 2, using a similar idea to that of [12]. In this way we manage to avoid the $O\left(\mathrm{~m}^{2}\right)$ term in the locating complexity of LZ-index, achieving the same locating time as [38], while reducing their space requirement of $(5+\epsilon) u H_{k}(T)+o(u \log \sigma)$ bits.

\subsection{Index Definition.}

We build basically on the LZ-index of Theorem 1, composed of LZTrie, RevTrie, and the $R$ mapping (compressed using suffix links $\varphi$ ). We add to LZTrie the data structure of Jansson et al. [21] to compute level ancestor queries, $L A(x, d)$, which gets the ancestor at depth $d$ of node $x$. This requires $o(n)$ extra bits and supports $L A$ queries in constant time. Therefore, the overall space requirement of the three above data structures is $(1+\epsilon) u H_{k}+o(u \log \sigma)$ bits.

To avoid the $O\left(\mathrm{~m}^{2}\right)$ term in the locating complexity, we should avoid occurrences of type 3 , since they make us check the $O\left(\mathrm{~m}^{2}\right)$ possible candidates. We cannot use the same procedure as for occurrences of type 2 (using the Range data structure) because LZTrie is only able to index whole phrases, and not text suffixes. Then, by using LZTrie to query the Range data structure we are only capable to get the phrases starting with a given suffix $P[i+1 . . m]$ of the pattern, an therefore we can find only occurrences spanning two consecutive phrases (i.e., occurrences of type 2).

Hence we add the alphabet friendly FM-index [13] of $T$ (AF-FMI(T) for short) to our index. By itself this self-index is able to search for pattern occurrences, requiring $u H_{k}(T)+o(u \log \sigma)$ bits of

space. However, its locate time per occurrence is $O\left(\log ^{1+\epsilon} u \frac{\log \sigma}{\log \log u}\right)$, for any constant $\epsilon>0$, which is greater than the $O(\log u)$ time per occurrence of LZ-indices.

As AF-FMI $(T)$ is based on the Burrows-Wheeler Transform [7] of $T$ (bwt(T) for short), it can be (conceptually) thought of as the suffix array $S A_{T}$ of $T$ (see Section 2.4). The AF-FMI $(T)$ indexes text suffixes. In particular, we will be interested in those suffixes which are aligned with the LZ78 phrase beginnings. By using this structure to query the Range data structure (instead of using LZTrie) we will be able to find those text suffixes which are aligned with LZ78 phrases and that have $P[i+1 . . m]$ as a prefix. Thus, $P[i+1 . . m]$ can span more than two consecutive phrases, and therefore we will consider occurrences of type 3 as a special case of occurrences of type 2 .

To find occurrences spanning several phrases we re-define Range, the data structure for 2dimensional range searching. Now it will operate on the grid $[1 . . u] \times[1 . . n]$. For each LZ78 phrase with identifier $i d$, for $0<i d \leqslant n$, assume that the RevTrie node for $i d$ has preorder $j^{\prime}$, and that 
phrase $(i d+1)$ starts at position $p$ in $T$. Then we store the point $\left(i^{\prime}, j^{\prime}\right)$ in Range, where $i^{\prime}$ is the lexicographic order of the suffix of $T$ starting at position $p$, i.e. $S A_{T}\left[i^{\prime}\right]=p$ holds.

Suppose that we search for a given string $s_{2}$ in $\operatorname{AF}-F M I(T)$ and get the interval $\left[i_{1}, i_{2}\right]$ in the $\operatorname{bwt}(T)$ (equivalently, in the suffix array of $T$ ), and that the search for string $s_{1}^{r}$ in RevTrie yields a node such that the preorder interval for its subtree is $\left[j_{1}, j_{2}\right]$. Then, a search for $\left[i_{1}, i_{2}\right] \times\left[j_{1}, j_{2}\right]$ in Range yields all phrases ending with $s_{1}$ such that the next phrase is aligned with an occurrence of $s_{2}$ in $T$.

We transform the grid $[1 . . u] \times[1 . . n]$ indexed by Range to an equivalent grid $[1 . . n] \times[1 . . n]$ by defining a bit vector $V[1 . . u]$, which indicates (with a 1) which positions of $\operatorname{AF}-\mathrm{FMI}(T)$ point to an LZ78 phrase beginning. We represent $V$ with the data structure of [36] allowing rank queries, and requiring $u H_{0}(V)+o(u) \leqslant n \log \frac{u}{n}+o(u) \leqslant \frac{u \log \log u}{\log _{\sigma} u}+o(u)=o(u \log \sigma)$ bits of storage. Thus, instead of storing the point $\left(i^{\prime}, j^{\prime}\right)$ as in the previous definition of Range, we store the point $\left(r a n k_{1}\left(V, i^{\prime}\right), j^{\prime}\right)$. The same search of the previous paragraph now becomes $\left[\operatorname{rank}_{1}\left(V, i_{1}\right), \operatorname{rank}_{1}\left(V, i_{2}\right)\right] \times\left[j_{1}, j_{2}\right]$.

As there is only one point per row and column of Range, we can use the data structure of Chazelle [8], which can be implemented by using $n \log n+O(n \log \log n)=u H_{k}(T)+o(u \log \sigma)$ bits [25]. As a result, the overall space requirement of our LZ-index is $(3+\epsilon) u H_{k}(T)+o(u \log \sigma)$, for any $k=o\left(\log _{\sigma} u\right)$ and any $0<\epsilon<1$.

\subsection{Search Algorithm}

For exists and count queries we can achieve $O(m)$ time by just using the $\operatorname{AF}-\mathrm{FMI}(T)$. We focus now on locate queries. Assume that $P[1 . . m]=p_{1} \ldots p_{m}$, for $p_{i} \in \Sigma$. As explained, we need to consider only occurrences of $P$ in $T$ of type 1 and 2 . Those of type 1 are solved just as for the original LZ-index, in $O\left(m+o c c_{1}\right)$ time. The rest of the section is devoted to those of type 2.

To find the pattern occurrences spanning two or more consecutive phrases we must consider the $m-1$ partitions $P[1 . . i]$ and $P[i+1 . . m]$ of $P$, for $1 \leqslant i<m$. For every partition we must find all phrases terminated with $P[1 . . i]$ such that the next phrase starts at the same position as an occurrence of $P[i+1 . . m]$ in $T$. Hence, as explained before, we must search for $P^{r}[1 . . i]$ in RevTrie and for $P[i+1 . . m]$ in $\operatorname{AF}-\mathrm{FMI}(T)$. Thus, every partition produces two one-dimensional intervals, one in each of the above structures.

If the search in RevTrie for $P^{r}[1 . . i]$ yields the preorder interval $\left[j_{1}, j_{2}\right]$, and the search for $P[i+$ 1..m] in $\operatorname{AF}-\mathrm{FMI}(T)$ yields interval $\left[i_{1}, i_{2}\right]$, the two-dimensional $\operatorname{range}\left[\operatorname{rank}_{1}\left(V, i_{1}\right), \operatorname{rank}_{1}\left(V, i_{2}\right)\right] \times$ $\left[j_{1}, j_{2}\right]$ in Range yields all pattern occurrences for the given partition of $P$. For every pattern occurrence we get a point $\left(i^{\prime}, j^{\prime}\right)$ from Range. The corresponding phrase identifier can be found as $t=i d s\left(R\left(j^{\prime}\right)\right)$, to finally report a pattern occurrence $\llbracket t, i \rrbracket$.

Overall, occurrences of type 2 are found in $O\left(\left(m+o c c_{2}\right) \log n\right)$ time. Yet, we still have to show how to find efficiently the intervals inf $\operatorname{AF}-F M I(T)$ and in RevTrie.

The $m-1$ intervals for $P[i+1 . . m]$ in $\operatorname{AF}-\operatorname{FMI}(T)$ can be found in $O(m)$ time thanks to the backward search concept, since the process to count the number of occurrences of $P[2 . . m]$ proceeds in $m-1$ steps, each one taking constant time if $\sigma=O(\operatorname{polylog}(u))$ [12]: in the first step we find the BWT interval for $p_{m}$, then we find the interval for occurrences of $p_{m-1} p_{m}$, then $p_{m-2} p_{m-1} p_{m}$, and so on to finally find the interval for $p_{2} \ldots p_{m}=P[2 . . m]$.

However, the work in RevTrie can take time $O\left(m^{2}\right)$ if we search for strings $P^{r}[1 . . i]$ separately, as done for the indices of Section 4. Fortunately, some work done to search for a given $P^{r}[1 . . i]$ can be reused to search for other strings. We have to search for strings $p_{m-1} p_{m-2} \ldots p_{1} ; p_{m-2} \ldots p_{1} ; \ldots$; and $p_{1}$ in RevTrie. Note that every $p_{j} \ldots p_{1}$ is the longest proper suffix of $p_{j+1} p_{j} \ldots p_{1}$. Suppose 
that we successfully search for $P^{r}[1 . . m-1]=p_{m-1} p_{m-2} \ldots p_{1}$, reaching the node with preorder $i^{\prime}$ in RevTrie, hence finding the corresponding preorder interval in RevTrie in $O(m)$ time. Now, to find the node representing suffix $p_{m-2} \ldots p_{1}$ we only need to follow suffix link $\varphi\left(i^{\prime}\right)$ (which takes $O(1)$ time) instead of searching for it from the RevTrie root (which would take $O(m)$ time again). The process of following suffix links can be repeated $m-1$ times up to reaching the node corresponding to string $p_{1}$, with total time $O(m)$. This is the main idea to get the $m-1$ preorder intervals in RevTrie in time less than quadratic. The general case is slightly more complicated and corresponds to the descend and suffix walk method used in [38].

In the sequel we explain the way we implement descend and suffix walk in our data structure. However, we must prove a couple of properties for RevTrie in order to be able to apply this method. First, we know that every non-empty node in RevTrie has a suffix link (see Lemma 4), yet we need to prove that every RevTrie node (including empty-non-unary nodes) has also a suffix link.

Lemma 8. Every empty non-unary node in RevTrie has a suffix link.

Proof. Assume that node $v_{r}$ in RevTrie is empty non-unary, and that it represents string ax, for $a \in \Sigma$ and $x \in \Sigma^{*}$. As node $v_{r}$ is empty non-unary, the node has at least two children. In other words, there exist at least two strings of the form axy and $a x z$, for $y, z \in \Sigma^{*}, y \neq z$, both strings corresponding to non-empty nodes, and hence these nodes have a suffix link. These suffix links correspond to strings $x y$ and $x z$ in RevTrie. Thus, there must exist a non-unary node for string $x$, which is the suffix link of node $v_{r}$.

The descent process in RevTrie will be a little bit different from the one described in the proof of Lemma 3. This time, we are going to reuse the work done for a string already searched in RevTrie, so we have to be sure that every time we arrive to a RevTrie node, the string represented by that node match the corresponding pattern prefix (the usual skipping process of a Patricia tree does not ensure that). Thus, the second property is that, although RevTrie is a Patricia tree and hence we store only the first symbol of each edge label, we can get all of it.

Lemma 9. Any edge label of length $l$ in RevTrie can be extracted in $O(l)$ time.

Proof. Assume that we are at node $v_{r}$ in RevTrie, and want to extract the label for edge $e_{v_{r} v_{r}^{\prime}}$ between nodes $v_{r}$ and $v_{r}^{\prime}$ in RevTrie. Since we arrive at a node in RevTrie by descending from the root, the length of the string represented by a given node can be computed by summing up the skips we have seen in the descent. Let $l_{v_{r}}$ and $l_{v_{r}^{\prime}}$ be the length of strings representd by nodes $v_{r}$, and $v_{r}^{\prime}$ respectively. Then, $l_{v_{r}^{\prime}}-l_{v_{r}}$ is the length of the label of edge $e_{v_{r} v_{r}^{\prime}}$.

If we assume that node $v_{r}^{\prime}$ has preorder $j_{1}$ in RevTrie, we can access the LZTrie node from where to start the extraction of the label by $v_{l z}^{\prime}=L A\left(R\left[j_{1}\right], l_{v_{r}^{\prime}}-l_{v_{r}}\right)$, in constant time [21]. The label of $e_{v_{r} v_{r}^{\prime}}$ is the label of the $v_{l z}^{\prime}$-to-root path. Notice that with the level-ancestor query on LZTrie we avoid to extract the string represented by node $v_{r}$ in RevTrie, as it has been already extracted before descending to $v_{r}$.

In case that $v_{r}^{\prime}$ is an empty node, recall that the corresponding value $R\left[j_{1}\right]$ gets undefined. However, just as in the proof of Lemma 3, we can use the preorder of any non-empty node within the subtree of $v_{r}^{\prime}$ to map to the LZTrie. We compute the preorder of the next non-empty node within the subtree of $v_{r}^{\prime}$ as $j_{2}=\operatorname{select}_{1}\left(B \operatorname{rank}_{1}\left(B, j_{1}\right)+1\right)$, then the length of the corresponding string can be computed as depth $\left(R\left[j_{2}\right]\right.$ ) in LZTrie (this is because preorder $j_{2}$ corresponds to a 
non-empty node in RevTrie), and we compute $v_{l z}^{\prime}=L A\left(R\left[j_{2}\right]\right.$, $\left.\operatorname{depth}\left(R\left[j_{2}\right]\right)-l_{v_{r}}\right)$, to finally extract the edge label by moving to the parent $l_{v_{r}^{\prime}}-l_{v_{r}}$ times.

Thus, we search RevTrie as in a normal trie, comparing every symbol as we descend, without skipping as it is done in Lemma 3. In this way, every time we arrive to a RevTrie node, the string represented by that node will match the corresponding prefix of the pattern.

Previously we showed that it is possible to search for all strings $P^{r}[1 . . i]$ in time $O(m)$, assuming that $P^{r}[1 . . m-1]$ exists in RevTrie (therefore all $P^{r}[1 . . i]$ exist in RevTrie). The general case is as follows.

Let $P^{r}[1 . . m-1]=p_{m-1} \ldots p_{1}$ be the longest string that we need to search for in RevTrie. We define three integer indices on $P^{r}[1 . . m-1]$, which guide the search:

$i_{1}$ : marks the beginning of the pattern suffix we are currently searching for. It is initialized at 1 since we start searching for $p_{m-1} p_{m-2} \ldots p_{1}$;

$i_{2}$ : indicates the current symbol in the pattern which is being compared with a symbol in an edge

label, with the aim of descending to a child of the current node. Notice that $P^{r}\left[i_{1} . . i_{2}-1\right]$ is the part of the current pattern which has been matched with the edge labels of RevTrie; and

$i_{3}$ : delimits the string corresponding to the current node, which represents string $P^{r}\left[i_{1} . . i_{3}\right]$ in RevTrie. Thus $P^{r}\left[i_{3}+1 . . i_{2}-1\right]$ will be the part of the pattern which has been compared with the label of the edge leading to the node we are trying to descend.

Our descend and suffix walk will be composed of three basic operations: descend, suffix, and retraverse.

Descend. We start searching for $p_{m-1} p_{m-2} \ldots p_{1}$ from the RevTrie root, using the method of Lemma 9 and using $i_{2}$ to indicate the current symbol being compared in the descent. Every time we can descend to a non-empty-unary child node (after matching all the characters of an edge), we set $i_{3} \leftarrow i_{2}$ and continue descending in the same way from this node. If when trying to descend to a child node we find and empty-unary node (which were added because of the skips in RevTrie, see Section 3.4), the index $i_{3}$ is not updated as explained before. In this case, we continue the descent with $i_{2}$ from the empty-unary node, using Lemma 9.

Suffix. Now assume that, being at current node $v_{r}$ (with preorder $j_{1}$ in RevTrie and representing string $a x$, for $a \in \Sigma, x \in \Sigma^{*}$ ), we cannot descend to a child node $v_{r}^{\prime}$ (with preorder $j_{2}$ in RevTrie and representing string axyz, for $y, z \in \Sigma^{*}$, such that $|y z|>0$ ). Let $e_{v_{r} v_{r}^{\prime}}$ be the edge between nodes $v_{r}$ and $v_{r}^{\prime}$, with label $y z$, where $y=P\left[i_{3}+1 . . i_{2}-1\right]$ and $P^{r}\left[i_{2}\right] \neq z_{1}$. Thus we cannot descend to $v_{r}^{\prime}$ this means that $P^{r}\left[i_{2}\right] \neq z_{1}$, and hence there are no phrases ending with $P^{r}\left[1 . . i_{1}\right]$.

Then, we go on to consider the next suffix $P^{r}\left[1 . . i_{1}+1\right]$. To reuse the work done up to node $v_{r}$ (i.e. $P^{r}\left[i_{1} . . i_{3}\right]=a x$ ), we follow the suffix link to get the node $\varphi\left(j_{1}\right)$ representing string $x$, setting $i_{1} \leftarrow i_{1}+1$

Retraverse. We have reused the work up to $x$, but we had actually worked up to $x y$. Notice that suffix $x y$ exists for sure in RevTrie, yet it could be represented by an empty unary node which has been compressed in an edge. Therefore, from node $\varphi\left(j_{1}\right)$ we descend using $y=P^{r}\left[i_{3}+1 . . i_{2}-1\right]$. The edge $e_{v_{r} v_{r}^{\prime}}=y z$ could be split into a path of several nodes between nodes $\varphi\left(j_{1}\right)$ and $\varphi\left(j_{2}\right)$. As substring $y$ has been already checked in the previous step, the descent from node $\varphi\left(j_{1}\right)$ is done by skipping and checking only the first symbols of the edge labels (advancing $i_{3}$ accordingly as we reach 
new nodes). If being at node $v_{r}^{\prime \prime}$ and trying to descend to the next node we find an empty-unary node, we directly jump to the position of the next non-empty-unary node (with preorder $j_{3}$ ) and then compute the length $l$ of the string represented by that node.

We need here a bit vector $E$ marking the empty-unary nodes, in preorder. We preprocess $E$ with a data structure for rank and select queries, so this requires $n^{\prime}+o\left(n^{\prime}\right)$ extra bits. The node with preorder $j_{3}$ can be found by using rank and select on $E$. The length $l$ can be computed as the sum of the length of the current node plus $n_{e} \cdot \log u$, where $n_{e}$ is the number of empty-unary nodes (which have been explicitly represented in the DFUDS of the tree) between the current node and the one with preorder $j_{3}$ (which can be computed as the number of 1 s between the corresponding positions in $E$ ), and $\log u$ comes from the skips of empty-unary nodes (recall Section 3.4). In case that $l>|x y|$, we resume the suffix mode from $v_{r}^{\prime \prime}$. Otherwise we resume the retraverse mode from the node with preorder $j_{3}$. This process is carried out up to fully consuming string $y$, and then we resume the descend mode from the corresponding node.

After we find the first suffix $P^{r}[1 . . i]$ in RevTrie (if any), then we are sure that every suffix of it also exists in RevTrie (because this trie is suffix closed). The nodes corresponding to these suffixes are found by following suffix links.

Lemma 10. Given a string $P$ of length $m$, we can search for strings $P^{r}[1 . . i]$, for $1 \leqslant i<m$, in RevTrie in $O(m)$ time.

Proof. Consider the method just described. Indices $i_{1}, i_{2}$, and $i_{3}$ grow from 1 to at most $m$. For every constant-time action we carry out, at least one of those indexes increases. Thus the total work is $O(m)$.

Therefore, we have proved:

Theorem 3. There exists a compressed full-text self-index requiring $(3+\epsilon) u H_{k}(T)+o(u \log \sigma)$ bits of space, for $\sigma=O(\operatorname{polylog}(u))$, any $k=o\left(\log _{\sigma} u\right)$, and any $0<\epsilon<1$, which is able to: report the occ occurrences of pattern $P[1 . . m]$ in text $T[1 . . u]$ in $O\left(\left(m+\frac{o c c}{\epsilon}\right) \log u\right)$ worst-case time; count pattern occurrences in $O(m)$ time; and determine whether pattern $P$ exists in $T$ in $O(m)$ time.

\section{Optimal Displaying of Text Substrings}

As we said before, LZ-index is able to report occurrences in the format $\llbracket t$, offset $\rrbracket$, where $t$ is the phrase in which the occurrence starts and off set is the distance between the beginning of the occurrence and the end of the phrase, and therefore so are our indices of Sections 4, 5, and 6 . However, we can report occurrences as text positions by adding a bit vector TPos[1..u] that marks the $n$ phrase beginnings. Given a text position $i$, then $\operatorname{rank}_{1}(T p o s, i)$ is the phrase number $i$ belongs to. Given a phrase identifier $j$, select ${ }_{1}(T P o s, j)$ yields the text position at which the $j$-th phrase starts. Therefore, given an occurrence in the format $\llbracket t$, off set $\rrbracket$, the text position for that occurrence can be computed as select $_{1}(T P o s, t+1)$ - off set.

Such TPos can be represented with $u H_{0}($ TPos $)+o(u) \leqslant n \log \frac{u}{n}+o(u) \leqslant \frac{u \log \log u}{\log _{\sigma} u}+o(u)=$ $o(u \log \sigma)$ bits [36] (recall $\left.n \leqslant u / \log _{\sigma} u\right)$.

The algorithm for extract queries (of whole LZ78 phrases) described in Section 3.3 can be also used on the indices of Theorems 1,2, and 3, yet this time providing the text positions from where to extract (rather than the phrase identifiers), since these positions can be transformed into phrase identifiers by using data structure TPos. As the Node data structure is simulated by using $i d s^{-1}$, 
it takes $O\left(\ell\left(1+\frac{1}{\epsilon \log _{\sigma} \ell}\right)\right)$ time to extract any text substring of length $\ell$, since we perform $\ell$ parent operations to get the $\ell$ symbols we want to display, and we must pay $O(1 / \epsilon)$ to use $i d s^{-1}$ each time we go on to extract the next phrase, which in the (very) worst case is done $O\left(\ell / \log _{\sigma} \ell\right.$ ) times.

To extract the text with $x b w$-based LZ-index of Section 5, we use TPos to transform the text positions into phrase identifiers, and then we use $i d s^{-1}$ to find the preorder position of the corresponding phrase, to finally map to the $x b w$ representation of LZTrie by using Pos $^{-1}$ in $O\left(1 / \epsilon^{2}\right)$ time. Then we move to the parent in the $x b w$, displaying the corresponding symbol stored in $S_{\alpha}$. When we reach the tree root, we use $i d s^{-1}$ again to consider the next phrase, and map to the $x b w$ again. The time is therefore $O\left(\ell\left(1+\frac{1}{\epsilon^{2} \log _{\sigma} \ell}\right)\right)$.

The restriction of displaying only whole phrases can be avoided by adding a data structure for level-ancestor queries on LZTrie. The data structure defined in [21] builds on DFUDS, allows constant time computation of level-ancestor queries, and requires $o(n)$ extra bits of space. Thus, the part of a phrase that we do not need to display is skipped by using the appropriate level-ancestor query. Yet, the displaying time is not optimal, since we work $O(1)$ per extracted symbol and on a RAM we are able to handle $\Theta(\log u)$ bits per access, which means $\Theta(\log u / \log \sigma)=\Theta\left(\log _{\sigma} u\right)$ symbols per access.

Instead we will describe a technique that can be plugged to any of the indices proposed in Sections 4,5 , and 6 , for displaying any text substring $T[i . . i+\ell-1]$, in optimal $O\left(1+\ell / \log _{\sigma} u\right)$ time. A compressed data structure [40] to display any text substring of length $\Theta\left(\log _{\sigma} u\right)$ in constant time, turns out to have similarities with LZ-index. We take advantage of this similarity to plug it within our indices, with some modifications, and obtain improved time to display text substrings. In [40], they added auxiliary data structures of $o(u \log \sigma)$ bits to LZTrie to support this operation efficiently. Given a position $i$ of the text, we first find the phrase including the position $i$ by using $\operatorname{rank}_{1}($ TPos, $i$ ), then find the node of LZTrie that corresponds to the phrase using Node (that is, the corresponding implementation of it). Then displaying a phrase is equivalent to outputting the path going from the node to the root of LZTrie. The auxiliary data structure, of size $O(n \log \sigma)=$ $o(u \log \sigma)$ bits, permits outputting the path by chunks of $\Theta\left(\log _{\sigma} u\right)$ symbols in $O(1)$ time per chunk. In addition, it can also display not only whole phrases, but any text substring within this complexity. The reason is that any prefix of a phrase is also a phrase, and it can be found in constant time by using a level-ancestor query [21] on the LZTrie, requiring just $o(n)$ extra bits. Thus the displaying can start backward from anywhere in a phrase, and of course it can stop at any point as well.

We modify this method to plug it into our indices. In their original method [40], if more than one consecutive phrases have length less than $\left(\log _{\sigma} u\right) / 2$ each, their phrase identifiers are not stored. Instead the substring of the text including those phrases are stored without compression. This guarantees efficient displaying operation without increasing the space requirement. However this will cause the problem that we cannot find patterns including those phrases. Therefore in our modification we store both the phrases themselves and their phrase identifiers. The search algorithm remains as before. To decode short phrases we can just output the explicitly stored substring including the phrases. For each phrase with length at most $\left(\log _{\sigma} u\right) / 2$, we store a substring of length $\log u$ containing the phrase. Because there are at most $O(\sqrt{u})$ such phrases, we can store all these substrings in $O(\sqrt{u} \log u)=o(u)$ bits. These auxiliary structures work as long as we can convert a phrase identifier into a preorder position in LZtrie (that is, compute $i d s^{-1}$ ) in constant time. Hence they can be applied to all the data structures in Sections 4, 5, and 6 . 
Theorem 4. The indices of Theorem 1 and Theorem 2 (and also those of Sections 5 and 6) can be adapted to extract a text substring of length $\ell$ surrounding any text position in optimal $O\left(1+\frac{\ell}{\epsilon \log _{\sigma} u}\right)$ worst-case time, using only $o(u \log \sigma)$ extra bits of space, for any $0<1<\epsilon$.

\section{Handling Larger Alphabets}

For simplicity, throughout this paper we have assumed $\sigma=O(\operatorname{polylog}(u))$, or equivalently $\log \sigma=$ $O(\log \log u)$. Here we study the cases $\log \sigma=o(\log u)$ and $\log \sigma=\Theta(\log u)$.

\subsection{The Case $\log \sigma=o(\log u)$}

As long as $\log \sigma=o(\log u)$ holds, we can still have $k=o\left(\log _{\sigma} u\right)>0$, while it also holds that $n \log n=u H_{k}(T)+o(u \log \sigma)$ [24]. Therefore, the space requirements of the indices of Theorems 1 to 3 stay the same.

Index of Section 4. The data structure of [13], which we use to represent letts, has a time complexity of $O\left(\frac{\log \sigma}{\log \log u}\right)$ for rank and select queries; thus, we lose the constant time for operation $\operatorname{child}(x, \alpha)$ on the tries, which would increase the time complexity of the whole index. Yet, we can represent letts with the (more complicated) data structure used in [6], thus ensuring constant time for child $(x, \alpha)$ for any $\sigma$ and retaining the same time complexity in our theorems. None of the remaining data structures of the index are affected by the alphabet size. As a result, Theorem 2 can be extended for the case $\log \sigma=o(\log u)$, rather than only for $\sigma=O(\operatorname{polylog}(u))$.

Index of Section 5. The times for the operations on the $x b w$ representation of LZTrie are affected by the alphabet size, depending on the representation used for $S_{\alpha}$. If we use the data structure of Golynski et al. [16], occurrences of type 1 are found in $O\left(m \log \log \sigma+\frac{o c c}{\epsilon}\right)$ time, because of the subpath query we perform on LZTrie; occurrences of type 2 are found in $O\left(m^{2} \log \log \sigma+\frac{m}{\epsilon}+\right.$ $(m+$ occ $) \log n)$ time, where the first term comes from searching for the $m-1$ partitions of $P$ in $x b w$; and occurrences of type 3 are found in $O\left(m^{2} \log \log \sigma+\frac{m^{2}}{\epsilon^{2}}\right)$, where the first term comes from searching for the $O\left(\mathrm{~m}^{2}\right)$ pattern substrings in the $x b w$ representation of LZTrie. Overall, the time for locate is $O\left(m^{2}\left(\frac{1}{\epsilon^{2}}+\log \log \sigma\right)+(m+o c c) \log u\right)$. We can also replace $O(\log \log \sigma)$ for $O\left(\frac{\log \sigma}{\log \log u}\right)$ in all these figures.

Index of Section 6. For this index the only affected part is the Alphabet-Friendly FM-index, AF$\operatorname{FMI}(T)$, which still has a space requirement of $u H_{k}(T)+o(u \log \sigma)$ bits of space. The counting time is increased to $O\left(m\left(1+\frac{\log \sigma}{\log \log u}\right)\right)$. Thus, the time for locate of this version of LZ-index now becomes $O\left(m\left(1+\frac{\log \sigma}{\log \log u}\right)+\left(m+\frac{o c c}{\epsilon}\right) \log u\right)$, which is still $O\left(\left(m+\frac{o c c}{\epsilon}\right) \log u\right)$, the same as stated by Theorem 3 , since $m \frac{\log \sigma}{\log \log u}=O(m \log u)$. The counting time, on the other hand, now becomes $O\left(m\left(1+\frac{\log \sigma}{\log \log u}\right)\right)$. Thus, we have a more general version of Theorem 3 :

Theorem 5. There exists a compressed full-text self-index requiring $(3+\epsilon) u H_{k}(T)+o(u \log \sigma)$ bits of space, for any $k=o\left(\log _{\sigma} u\right)$, any $0<\epsilon<1$, and such that $\log \sigma=o(\log u)$, which is able to: report the occ occurrences of pattern $P[1 . . m]$ in text $T[1 . . u]$ in $O\left(\left(m+\frac{o c c}{\epsilon}\right) \log u\right)$ worst-case time; count pattern occurrences in $O\left(m\left(1+\frac{\log \sigma}{\log \log u}\right)\right)$ time; determine whether pattern $P$ exists in $T$ in $O\left(m\left(1+\frac{\log \sigma}{\log \log u}\right)\right)$ time; and extract any text substring of length $\ell$ in time $O\left(\ell /\left(\epsilon \log _{\sigma} u\right)\right)$. 


\subsection{The Case $\log \sigma=\Theta(\log u)$}

For the case $\log \sigma=\Theta(\log u)$, because of Lemma 1 we have that $n \log n=u H_{k}(T)+O(u(1+k \log \sigma))$ bits of space, which is $\Theta(u \log \sigma)$ even for $k=1$. Thus, high-order compression is lost. For $k=0$ the space is $u H_{0}(T)+o(u \log \sigma)$ bits of space, so zero-order compression is retained. On the other hand, all the time complexities obtained for the case $\log \sigma=o(\log u)$ are valid for this larger $\sigma$.

On the other hand, it has been shown that the empirical-entropy model is not so adequate for such a large alphabet [15].

\section{Conclusions and Future Work}

We have improved the overall performance of LZ-indices, achieving stronger compressed self-indices based on the Lempel-Ziv compression algorithm [42]. We have reduced the space of Navarro's LZindex [34] to about a half, achieving $(2+\epsilon) u H_{k}(T)+o(u \log \sigma)$ bits of space to index a text $T[1 . . u]$ with $k$-th order empirical entropy $H_{k}$, for any $k=o\left(\log _{\sigma} u\right)$ and any $0<\epsilon<1$. Therefore, ours is the smallest compressed self-index based on Lempel-Ziv compression. Our indices are able to search for the occ occurrences of a pattern $P[1 . . m]$ in $T$ in $O\left(\frac{m^{2}}{\epsilon^{2}}+(m+o c c) \log u\right)$ worst-case time, as well as extracting any text substring of length $\ell$ in optimal $O\left(\frac{\ell}{\epsilon \log _{\sigma} u}\right)$ time. Thus, we achieve the same locate time as the index of Kärkkäinen and Ukkonnen [23], yet with a much smaller index which does not need the text to operate. We also showed how the space can be squeezed to $(1+\epsilon) u H_{k}(T)+o(u \log \sigma)$ bits, with $O\left(m^{2}\right)$ average-case search time if $m \geqslant 2 \log _{\sigma} n$. This space approaches as much as desired the optimal $u H_{k}(T)$ under the $k$-th order empirical entropy model for all $k$. However, this index does not provides worst-case guarantees at search time.

We also showed how to use an LZ-index to achieve $O((m+o c c) \log u)$ time to locate the pattern occurrences, requiring $(3+\epsilon) u H_{k}(T)+o(u \log \sigma)$ bits of space. This is about a half of the space required by other LZ-indices having the same search time.

Thus, we have achieved LZ-indices with space requirements ranging from $(1+\epsilon)$ to $(3+\epsilon)$ times the empirical entropy of the text (plus lower-order terms), with different achievements in the time complexities according with the space requirement of the index. These indices are very competitive with state-of-the-art indices, both in time and space requirement.

The most basic problems for compressed self-indices are that of searching and reproducing the text. However, there are many other functionalities that a self-index must provide in order to be fully useful, as for example the space-efficient construction of the indices, secondary-memory capabilities (in case that the text is so huge that the corresponding compressed self-index does not fit in main memory), dynamic capabilities, and allowing more complex queries on the text (such as regular-expression and approximate searching).

Constructing the indices with little space is an important research topic, regarding the practicality of the indices $[20,19,2,26]$. For instance, it has been shown that the original LZ-index can be constructed using about the same space as the final index, both in theory and in practice. An interesting future work is to adapt these algorithms to our reduced-space variants. Also, it has been shown that the LZ-index can be efficiently handled on secondary storage [3], by means of adding extra redundancy to the index to avoid most random accesses. This provides a very promising alternative, yet an interesting question is whether we can use techniques similar to those of this paper to reduce the extra redundancy added to the index. It has been also shown that the LZ-indices (in particular the ILZI of Russo and Oliveira [38]) are adequate for approximate string matching [37]. 
Finally, a very important aspect is that of the practical implementations of compressed indices, as many theoretical indices are proposed but never implemented. The Pizza\&6Chili Corpus [14] provides practical implementations of compressed indices, as well as some example texts. To show the practicality of our approach, there are currently in the site some implementations of reduced schemes of LZ-index, based on ideas which are similar to the ones described in this paper. These indices have shown to be very competitive against others [10], specifically for locate and extract queries. We hope to get further results on this line.

\section{References}

1. A Apostolico. The myriad virtues of subword trees. In Combinatorial Algorithms on Words, NATO ISI Series, pages 85-96. Springer-Verlag, 1985.

2. D. Arroyuelo and G. Navarro. Space-efficient construction of LZ-index. In Proc. ISAAC, LNCS 3827, pages 1143-1152. Springer, 2005.

3. D. Arroyuelo and G Navarro. A Lempel-Ziv text index on secondary storage. In Proc. CPM, LNCS 4580, pages 83-94, 2007.

4. D. Arroyuelo, G. Navarro, and K. Sadakane. Reducing the space requirement of LZ-index. In Proc. CPM, LNCS 4009, pages 319-330, 2006.

5. J. Barbay, M. He, J. I. Munro, and S. S. Rao. Succinct indexes for strings, binary relations and multi-labeled trees. In Proc. SODA, pages 680-689, 2007.

6. D. Benoit, E. Demaine, J. I. Munro, R. Raman, V. Raman, and S. S. Rao. Representing trees of higher degree. Algorithmica, 43(4):275-292, 2005.

7. M. Burrows and D. J. Wheeler. A block-sorting lossless data compression algorithm. Technical Report 124, Digital Equipment Corporation, 1994.

8. B. Chazelle. A functional approach to data structures and its use in multidimensional searching. SIAM Journal on Computing, 17(3):427-462, 1988.

9. P. Elias. Universal codeword sets and representation of integers. IEEE Trans. Inform. Theory, 21(2):194-203, 1975.

10. P. Ferragina, R. González, G. Navarro, and R. Venturini. Compressed text indexes: From theory to practice! 2007. Submitted. http://pizzachili.dcc.uchile.cl/resources/cti_jea07.pdf.

11. P. Ferragina, F. Luccio, G. Manzini, and S. Muthukrishnan. Structuring labeled trees for optimal succinctness, and beyond. In Proc FOCS, pages 184-196, 2005.

12. P. Ferragina and G. Manzini. Indexing compressed text. Journal of the ACM, 54(4):552-581, 2005.

13. P. Ferragina, G. Manzini, V. Mäkinen, and G. Navarro. Compressed representations of sequences and full-text indexes. ACM Transactions on Algorithms (TALG), 3(2):article 20, 2007.

14. P. Ferragina and G. Navarro. Pizza\&Chili Corpus - Compressed indexes and their testbeds, 2005. http://pizzachili.dcc.uchile.cl.

15. T. Gagie. Large alphabets and incompressibility. Information Processing Letters, 99(6):246-251, 2006.

16. A. Golynski, J. I. Munro, and S. S. Rao. Rank/select operations on large alphabets: A tool for text indexing. In Proc. SODA, pages 368-373, 2006.

17. R. Grossi, A. Gupta, and J. S. Vitter. High-order entropy-compressed text indexes. In Proc. SODA, pages 841-850. SIAM, 2003.

18. R. Grossi and J. S. Vitter. Compressed suffix arrays and suffix trees with applications to text indexing and string matching. SIAM J. Comput., 35(2):378-407, 2005.

19. W. K. Hon, T. W. Lam, K. Sadakane, W.-K. Sung, and M. Yiu. A space and time efficient algorithm for constructing compressed suffix arrays. Algorithmica, 48(1):23-36, 2007.

20. W.-K. Hon, K. Sadakane, and W.-K. Sung. Breaking a time-and-space barrier in constructing full-text indices. In Proc. FOCS, pages 251-260, 2003.

21. J. Jansson, K. Sadakane, and W.-K. Sung. Ultra-succinct representation of ordered trees. In Proc. SODA, pages 575-584, 2007.

22. J. Kärkkäinen. Repetition-based text indexes. PhD thesis, Dept. of CS, University of Helsinki, Finland, 1999.

23. J. Kärkkäinen and E. Ukkonen. Lempel-Ziv parsing and sublinear-size index structures for string matching. In Proc. WSP, pages 141-155. Carleton University Press, 1996.

24. R. Kosaraju and G. Manzini. Compression of low entropy strings with Lempel-Ziv algorithms. SIAM Journal on Computing, 29(3):893-911, 1999. 
25. V. Mäkinen and G. Navarro. Rank and select revisited and extended. Theoretical Computer Science, 387(3):332$347,2007$.

26. V. Mäkinen and G. Navarro. Dynamic entropy-compressed sequences and full-text indexes. ACM Transactions on Algorithms (TALG), 2008. To appear.

27. U. Manber and G. Myers. Suffix arrays: A new method for on-line string searches. SIAM Journal on Computing, 22(5):935-948, 1993.

28. G. Manzini. An analysis of the Burrows-Wheeler transform. Journal of the ACM, 48(3):407-430, 2001.

29. D. R. Morrison. Patricia - practical algorithm to retrieve information coded in alphanumeric. Journal of the ACM, 15(4):514-534, 1968.

30. J. I. Munro. Tables. In Proc. FSTTCS, LNCS 1180, pages 37-42. Springer, 1996.

31. J. I. Munro, R. Raman, V. Raman, and S. S. Rao. Succinct representations of permutations. In ICALP, LNCS 2719, pages 345-356, 2003.

32. J. I. Munro and V. Raman. Succinct representation of balanced parentheses and static trees. SIAM Journal on Computing, 31(3):762-776, 2001.

33. G. Navarro. Indexing text using the Ziv-Lempel trie. Technical Report TR/DCC-2003-0, Dept. of Computer Science, Univ. of Chile, 2003. ftp://ftp.dcc.uchile.cl/pub/users/gnavarro/jlzindex.ps.gz.

34. G. Navarro. Indexing text using the Ziv-Lempel trie. Journal of Discrete Algorithms (JDA), 2(1):87-114, 2004.

35. G. Navarro and V. Mäkinen. Compressed full-text indexes. ACM Computing Surveys, 39(1):article 2, 2007.

36. R. Raman, V. Raman, and S. S. Rao. Succinct indexable dictionaries with applications to encoding $k$-ary trees and multisets. In Proc. SODA, pages 233-242, 2002.

37. L. Russo, G. Navarro, and A. Oliveira. Approximate string matching with Lempel-Ziv compressed indexes. In Proc. SPIRE, LNCS 4726, pages 264-275, 2007.

38. L. Russo and A. Oliveira. A compressed self-index using a Ziv-Lempel dictionary. In Proc. SPIRE, LNCS 4209, pages 163-180, 2006.

39. K. Sadakane. New Text Indexing Functionalities of the Compressed Suffix Arrays. Journal of Algorithms, 48(2):294-313, 2003.

40. K. Sadakane and R. Grossi. Squeezing Succinct Data Structures into Entropy Bounds. In Proc. SODA, pages 1230-1239, 2006.

41. E. Ukkonen. On-line construction of suffix trees. Algorithmica, 14(3):249-260, 1995.

42. J. Ziv and A. Lempel. Compression of individual sequences via variable-rate coding. IEEE Trans. Inform. Theory, 24(5):530-536, 1978. 